\title{
Pseudomonas syringae Two-Component Response Regulator RhpR Regulates Promoters Carrying an Inverted Repeat Element
}

\author{
Xin Deng, ${ }^{1}$ Lefu Lan,, ${ }^{1}$ Yanmei Xiao, ${ }^{1}$ Megan Kennelly, ${ }^{1}$ Jian-Min Zhou, ${ }^{2}$ and Xiaoyan Tang ${ }^{1,3}$ \\ ${ }^{1}$ Department of Plant Pathology, Kansas State University, Manhattan 66506-5502, U.S.A.; ${ }^{2}$ National Institute of Biological \\ Sciences, Beijing, China; ${ }^{3}$ Shenzhen Molecular Crop Design Center for Tropical and Subtropical Regions, Shenzhen, China
}

Submitted 21 October 2009. Accepted 20 March 2010.

\begin{abstract}
The two-component system RhpRS was identified in Pseudomonas syringae as a regulator of the genes encoding the type III secretion system and type III effector proteins (together called the $\mathrm{T} 3$ genes). In the absence of the sensor kinase $\mathrm{RhpS}$, the response regulator $\mathrm{RhpR}$ represses the induction of the $\mathrm{T3}$ gene regulatory cascade consisting of $h r p R S, h r p L$, and the $\mathrm{T3}$ genes in a phosphorylation-dependent manner. The repressor activity of $\mathrm{RhpR}$ is inhibited by $\mathrm{RhpS}$, which presumably acts as a phosphatase under the T3 gene inducing conditions. Here, we show that $R h p R$ binds and induces its own promoter in a phosphorylation-dependent manner. Deletion and mutagenesis analyses revealed an inverted re-

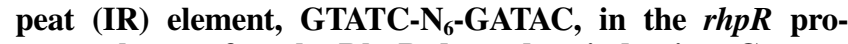
moter that confers the RhpR-dependent induction. Computational search of the $P$. syringae genomes for the putative IR elements and Northern blot analysis of the genes with a putative IR element in the promoter region uncovered five genes that were upregulated and two genes that were downregulated in an RhpR-dependent manner. Two genes that were strongly induced by $R h p R$ were assayed for the IR element activity in gene regulation and, in both cases, the IR element mediated the RhpR-dependent gene induction. Chromatin immunoprecipitation assays indicated that RhpR binds the promoters containing a putative IR element but not the $h r p R$ and $h r p L$ promoters that do not have an IR element, suggesting that $R h p R$ indirectly regulates the transcriptional cascade of $h r p R S, h r p L$, and the T3 genes.
\end{abstract}

Bacteria primarily rely on two-component systems (TCS) to sense and respond to environmental changes (Hoch 2000). A TCS usually consists of a sensor histidine kinase and a response regulator (Stock et al. 2000). In general, the sensor kinase, upon sensing a specific signal, autophosphorylates at a highly conserved histidine residue in the transmitter domain and, subsequently, transfers the phosphoryl group to an aspartate residue in the receiver domain of its cognate response regulator. Phosphorylation activates the response regulator which, in turn, stimulates or represses the transcription of its target genes (Stock et al. 2000). Many TCS sensor kinases also possess a phosphatase activity that can dephosphorylate the cognate response regulators and retain the later in an inactive state (Bijlsma and Groisman 2003). The relative kinase and phosphatase activities in bacterial cells are modulated by environmental stimuli and determine the outcome of signal trans-

Corresponding author: Xiaoyan Tang; Telephone: +1.785.532.1345; Fax: +1.785 .532 .5692 ; E-mail: xtang@ ksu.edu duction. Response regulators can be phosphorylated by unrelated sensor kinases or by small phosphate donor molecules such as acetyl phosphate in the absence of cognate sensor kinases (McCleary et al. 1993; Laub and Goulian 2007). As a result, many response regulators display a regulatory activity even in the absence of their cognate sensor kinases (Laub and Goulian 2007).

DNA-binding response regulators usually bind directly to promoter elements to mediate gene regulation. Most response regulators bind to DNA elements consisting of direct or inverted repeats (IR) that are separated by a spacer of 2 to 11 base nucleotides (de Been et al. 2008). For example, response regulators in the OmpR family typically bind to direct repeat elements separated by a spacer of four or five nucleotides, whereas response regulators of the NarL family usually bind to IR elements separated by two to six nucleotides (de Been et al. 2008). A direct repeat element, (T/G)GTTTA- $\mathrm{N}_{5}-(\mathrm{T} / \mathrm{G})$ GTTTA, is defined as the PhoP box (Groisman 2001), whereas an imperfect IR (GCGGC-N $\mathrm{N}_{5}$-GTCGC) is critical for DNA binding of the response regulator RegR of Bradyrhizobium japonicum (Emmerich et al. 2000). Response regulators are believed to form homodimers on the repeat elements (Blanco et al. 2002; Maris et al. 2002). Some promoters have several copies of the repeat elements, and response regulators often form an oligomer on such promoters (Maris et al. 2005).

Some response regulators are capable of positive as well as negative regulation of their target genes. For example, the phosphorylated response regulator OmpR (P-OmpR) of Escherichia coli binds the promoters of $o m p F$ and $o m p C$ genes and regulates their expression in response to medium osmolarity (Head et al. 1998; Lan and Igo 1998). There are four and three $\mathrm{P}-\mathrm{OmpR}$ binding sites in the promoters of ompF and ompC, respectively (Yoshida et al. 2006). At low osmolarity, P-OmpR binds to two or three high-affinity sites in the $o m p F$ promoter and activates $o m p F$. Under this condition, only one site in the ompC promoter is occupied by $\mathrm{P}-\mathrm{OmpR}$, which is insufficient to activate $\operatorname{ompC}$. At high osmolarity, P-OmpR occupies all three sites in the $o m p C$ promoter to activate $\operatorname{ompC}$ and all four sites in the $o m p F$ promoter to inhibit ompF (Yoshida et al. 2006). The response regulator CovR of Streptococcus pyogenes can also directly activate and repress its target genes (Churchward 2007). CovR represses its target genes via promoter occlusion, because the CovR binding site overlaps with the sigma 70 promoter or the transcriptional start site (Gao et al. 2005; Gusa and Scott 2005; Gusa et al. 2006). CovR activates the expression of the dipeptide permease gene $d p p A$ by interfering with the binding of a repressor protein to the $d p p A$ gene promoter (Gusa et al. 2007). 
In addition to regulating the downstream genes, many TCS response regulators are capable of self-regulation (Bijlsma and Groisman 2003). Self-regulation is mediated by direct binding of the response regulator protein to its own gene promoter (Bijlsma and Groisman 2003). Many response regulators autoactivate their expression (Soncini et al. 1995; Bang et al. 2002; Clarke and Sperandio 2005; Gonzalo-Asensio et al. 2008) but a few response regulators such as CovR and TorR are capable of autoinhibition (Ansaldi et al. 2000; Gusa and Scott 2005). Direct self-regulation enables bacteria to respond more rapidly and efficiently to environmental changes (Hoffer et al. 2001; Shin et al. 2006).

The genes encoding the type III secretion system and type III effector proteins (together called the T3 genes) of Pseudomonas syringae are repressed in rich medium but induced in the plant and minimal medium (MM) (Xiao et al. 2004; Tang et al. 2006). In searching for genes regulating the induction of the T3 genes, we identified the $\operatorname{rhp} S^{-}$mutation that severely inhibited the induction of the T3 genes in the plant and MM (Xiao et al. 2007; Deng et al. 2009). rhpS encodes a putative TCS sensor histidine kinase. $r h p S$ is downstream of $r h p R$ in an operon. $r h p R$ encodes a putative TCS response regulator. Deletion of the whole rhpRS locus $(\triangle r h p R S)$ restores the induction of the T3 genes, and overexpression of RhpR in the deletion mutant $\triangle r h p R S$ suppresses the induction of the $\mathrm{T} 3$ genes in a phosphorylation-dependent manner (Xiao et al. 2007). It appears that RhpR is phosphorylated by an as-yet-unknown factor in the $\operatorname{rhpS} S^{-}$mutant, the phosphorylated RhpR (P-RhpR) represses the T3 genes, and RhpS acts as a phosphatase and retains RhpR in a dephosphorylated state under conditions inducing the $\mathrm{T} 3$ genes.
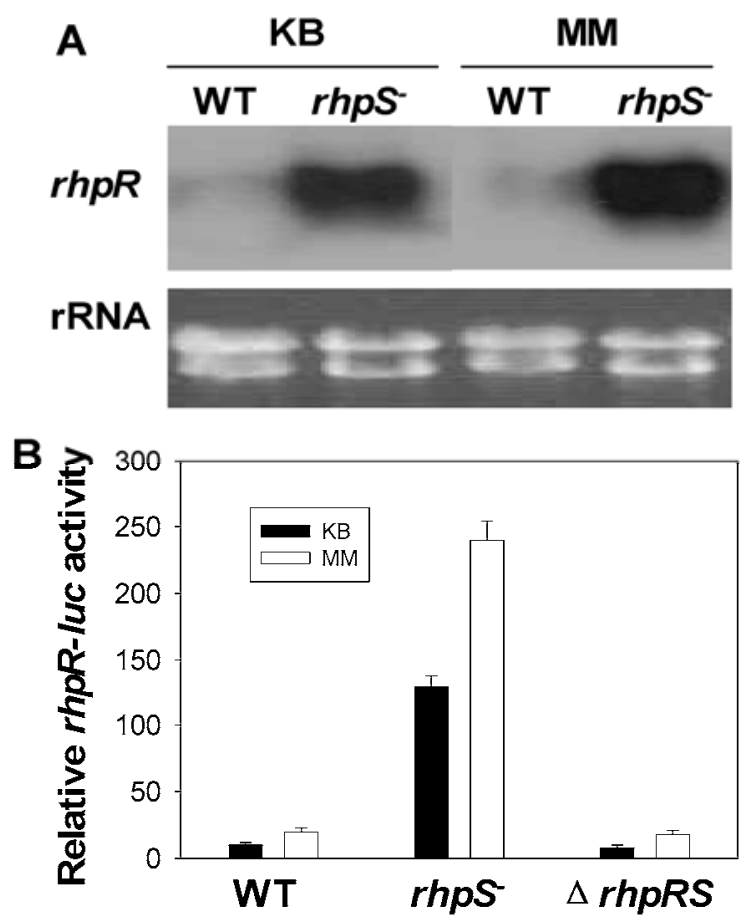

Fig. 1. $r h p R$ is highly induced in the $r h p S^{-}$mutant. A, Wild-type DC3000 (WT) and the rhps mutant were grown in King's B (KB) medium and subsequently induced in minimal medium (MM) for $6 \mathrm{~h}$ before RNA extraction. Total RNA ( $10 \mu \mathrm{g} / \mathrm{sample})$ was analyzed by RNA blotting with the radio-labeled $r h p R$ probes. The ethidium bromide-stained RNA gel indicates the loading of RNA samples. B, WT, the $\operatorname{rhpS} S^{-}$mutant, and the $\Delta r h p R S$ mutant carrying the pHM2::rhpR $R_{540^{-}}$luc reporter gene were cultured in $\mathrm{KB}$ medium and then induced in MM. The luciferase activities were measured at $0 \mathrm{~h}(\mathrm{~KB})$ and $6 \mathrm{~h}(\mathrm{MM})$ after induction in $\mathrm{MM}$ and normalized to bacterial numbers. Each data point represents the average of three measurements. Error bars indicate standard deviations.
Here, we show that RhpR binds and activates its own promoter in a phosphorylation-dependent manner. An IR element was found in the rhpR promoter that mediates the RhpRdependent regulation. Through genome-wide searching of the IR element-containing promoters and Northern blot analysis of the corresponding genes, putative IR element-regulated genes were identified.

\section{RESULTS}

The $r h p R$ promoter is induced by $R h p R$.

Our previous studies showed that RhpR represses the T3 genes in the $\operatorname{rhpS} S^{-}$mutant, and the presence of RhpS derepresses the T3 genes in MM (Xiao et al. 2007). In an attempt to depict the regulatory pathway, we searched for the RhpRregulated promoters. Bacterial TCS loci are often subject to direct autoregulation by the response regulators (Bijlsma and Groisman 2003). To determine whether RhpR regulates rhpRS expression, Northern hybridization was performed to compare the expression of $r h p R$ RNA in wild-type (WT) DC3000 and the transposon insertion mutant of the $r h p S$ gene. $r h p R$ RNA was expressed at a much higher level in the $\operatorname{rhp} S^{-}$mutant than in the WT strain in MM as well as in rich King's B (KB) medium (Fig. 1A).

To test whether RhpR regulates the activity of the $r h p R$ promoter, $540 \mathrm{bp}$ of $r h p R$ promoter DNA (including the start codon ATG of the rhpR gene) was fused to the promoterless luciferase (luc) reporter gene, and the resulting plasmid, pHM2::rhpR $R_{540}$-luc (Table 1), was introduced into WT DC3000, the $r h p S^{-}$mutant, and the $\triangle r h p R S$ mutant. Reporter activity was 10 -fold higher in the $\operatorname{rhp} S^{-}$mutant than in the WT and $\triangle r h p R S$ mutant strains (Fig. 1B), indicating an autoactivation of the $r h p R$ promoter by RhpR.

\section{RhpR requires the phosphorylation site to activate the $r h p R$ promoter.}

RhpR requires the phosphorylation site for repression of the T3 genes in P. syringae strains (Xiao et al. 2007). To determine whether the inducing activity of RhpR is also regulated by phosphorylation, the $\operatorname{RhpR}(\mathrm{D} 70 \mathrm{~A})$ mutant with the predicted phosphorylation site Asp70 substituted by alanine was tested for the activity to induce the $r h p R$ promoter. Hemagglutinin (HA)-tagged WT RhpR and RhpR(D70A) mutant proteins were expressed in the $\Delta r h p R S$ mutant using a constitutive promoter harbored by the pML122 plasmid (Table 1). Western blot analysis indicated that RhpR-HA and RhpR(D70A)-HA proteins were expressed at similar levels (Xiao et al. 2007). The expression of RhpR-HA but not RhpR(D70A)-HA in the $\Delta r h p R S$ mutant strongly induced the $r h p R_{540}$ promoter in both $\mathrm{MM}$ and $\mathrm{KB}$ (Fig. 2A), suggesting that phosphorylation of RhpR enhances its regulatory activity. These results also supported our hypothesis that RhpR is phosphorylated by unknown factors in the $r h p S^{-}$mutant (Xiao et al. 2007).

In addition to the RhpR-dependent induction, the $r h p R_{540^{-}}$ luc reporter gene in WT DC3000, the $\operatorname{rhpS} S^{-}$mutant, and the $\Delta r h p R S$ mutant displayed higher activities in $\mathrm{MM}$ than in $\mathrm{KB}$ medium (Figs. 1B and 2A), indicating an RhpR-independent induction of the $r h p R$ promoter by MM.

The RhpR-dependent induction of the $r h p R$ promoter led us to test whether RhpR directly regulates the $r h p R$ promoter. Our attempts to purify the recombinant RhpR protein from $E$. coli were unsuccessful, which deterred the in vitro assays of RhpR interaction with the $r h p R$ promoter. Thus, we tested whether the expression of the recombinant RhpR protein can induce the $r h p R$ promoter in E. coli cells. Plasmids expressing GST-RhpR and GST-RhpR(D70A) fusion proteins were transformed into E. coli BL21 carrying the pHM2::rhpR $R_{540^{-}}$luc plasmid. Consis- 
tent with the result in $\Delta r h p R S$ mutant (Fig. 2A), the $r h p R_{540^{-}}$ $l u c$ reporter gene displayed a higher activity in the BL21 strain expressing GST-RhpR protein than in the BL21 strain expressing GST-RhpR(D70A) (Fig. 2B), suggesting direct regulation of the $r h p R$ promoter by RhpR.

\section{RhpR requires the phosphorylation site for maximal association with the $r h p R$ promoter.}

A chromatin immunoprecipitation (ChIP) assay was performed to test whether RhpR directly binds the $r h p R$ promoter in $P$. syringae, and whether the binding activity of $\mathrm{RhpR}$ is affected by phosphorylation. $\Delta r h p R S$ mutant strains expressing RhpR-HA and RhpR(D70A)-HA proteins were used for ChIP assay. RhpR-HA and RhpR(D70A)-HA were expressed at similar levels in the $\Delta r h p R S$ mutant (Xiao et al. 2007). The $\Delta r h p R S$ mutant carrying the empty pML122 plasmid was used as a negative control. A ChIP assay was performed with the anti-HA antibodies, and the enrichment of the $r h p R$ promoter DNA in the immunocomplexes was detected using quantitative real-time polymerase chain reaction (qRT-PCR). The primer used for amplification of the $r h p R$ promoter DNA is listed in Table 2. A ChIP assay was also performed without the use of the anti-HA antibodies to determine nonspecific precipitation of the promoter DNA. The amount of the promoter DNA precipitated by the anti-HA antibodies subtracted by the amount of the promoter DNA derived from nonspecific precipitation was regarded as the enrichment of the promoter DNA by the anti-HA antibodies. The rhpR promoter DNA was enriched twofold by the anti-HA antibodies in the control strain carrying the empty pML122 vector (Fig. 3). In contrast, the $r h p R$ promoter DNA was enriched 215-fold in the strain expressing RhpR-HA (Fig. 3). The large enrichment of the $r h p R$ promoter DNA from the RhpRHA-expressing strain indicated a direct binding of RhpR-HA with the $r h p R$ promoter. The $r h p R$ promoter DNA was enriched only fivefold in the strain expressing RhpR(D70A)-HA (Fig. $3)$. The much larger enrichment of the $r h p R$ promoter DNA from the RhpR-HA-expressing strain than the RhpR(D70A)-HAexpressing strain (215- versus fivefold) was consistent with the strong induction of $r h p R$ promoter by $\mathrm{RhpR}$ but not by $\operatorname{RhpR}(\mathrm{D} 70 \mathrm{~A})$ in the $\Delta r h p R S$ mutant, suggesting that phosphorylation of RhpR increases its interaction with the rhpR promoter. The differential enrichment of the promoter DNA by the anti-HA antibodies was not detected in PSPTO1489 (Fig. 3), a housekeeping gene that is not regulated by RhpR (L. Lan and $\mathrm{X}$. Tang, unpublished data).

\section{Identification of the RhpR-regulated element in the $\operatorname{rhpR}$ promoter.}

The 540-bp $r h p R$ promoter in the pHM2::rhpR $R_{540}-l u c$ plasmid was deleted to $170,120,80$, and $40 \mathrm{bp}$ upstream of the $r h p R$ start codon ATG, and the resulting deletions were assayed for the promoter activities in the $r h p S^{-}$mutant (Fig. 4A). The 170bp promoter $\left(r h p R_{170}\right)$ had approximately $60 \%$ of the activity relative to $r h p R_{540}$. However, the 120 -bp promoter $\left(r h p R_{120}\right)$ had only $7 \%$ of the activity. Further deletion to 40 bp completely eliminated the promoter activity. $r h p R_{170}$ and $r h p R_{120}$ promoters displayed low activities at the same level in the $\Delta r h p R S$ mutant (data not shown). These indicated the presence of an RhpR-dependent promoter element in the region between -170 and -120 .

The region between -170 and -120 has a perfect 5-bp IR sequence (between -147 and -132) with a 6-bp spacer, GTATC$\mathrm{N}_{6}$-GATAC (Fig. 4C). To test whether this IR element has a role in the RhpR-dependent regulation, two additional deletions were generated in the region between -170 and -120 . The 147-bp promoter carrying the IR element displayed strong ac-

Table 1. Plasmids

\begin{tabular}{|c|c|c|}
\hline Plasmid name & Description & Reference \\
\hline pML122::rhpR-HA & $r h p R-H A$ in pML122 plasmid, under pNm promoter & Xiao et al. 2007 \\
\hline pML122::rhpR(D7OA)-HA & Derived from pML122::rhpR-HA, with Asp70 replaced by Ala & Xiao et al. 2007 \\
\hline pML122 & Broad-host plasmid & Labes et al. 1990 \\
\hline pHM2 & Broad-host plasmid & Xiao et al. 2007 \\
\hline pBluescript-SK-luc & firefly $l u c$ in pBluescript-SK(+) & Deng et al. 2009 \\
\hline pGEX-KG & plasmid to produce GST-fusion protein & Guan et al. 1991 \\
\hline pHM2-luc & Broad host plasmid for reporter genes construction & Xiao et al. 2007 \\
\hline $\mathrm{pHM} 2:: r h p R_{540}-l u c$ & rhpR-luc (-540 from ATG) reporter in pHM2 & This study \\
\hline pHM2:: $\operatorname{rhp} R_{170^{-}} l u c$ & rhpR-luc (-170 from ATG) reporter in pHM2 & This study \\
\hline pHM2:: $r h p R_{147^{-}} l u c^{*}$ & rhpR-luc (-147 from ATG) reporter in pHM2 & This study \\
\hline pHM2:: rhpR $R_{132-l u c}$ & rhpR-luc ( -132 from ATG) reporter in pHM2 & This study \\
\hline pHM2:: $r h p R_{120^{-l u c}}$ & rhpR-luc ( -120 from ATG) reporter in pHM2 & This study \\
\hline pHM2:: $r h p R_{80}-l u c$ & rhpR-luc (-80 from ATG) reporter in pHM2 & This study \\
\hline pHM2:: $r h p R_{40}-l u c$ & rhpR-luc ( -40 from ATG) reporter in pHM2 & This study \\
\hline pHM2::rhpR-pro-147GT-luc & Derived from $*$, with $-147 \mathrm{G}$ replaced by $\mathrm{T}$ & This study \\
\hline pHM2::rhpR-pro-146TG-luc & Derived from *, with $-146 \mathrm{~T}$ replaced by G & This study \\
\hline pHM2::rhpR-pro-145AC-luc & Derived from $*$, with $-145 \mathrm{~A}$ replaced by $\mathrm{C}$ & This study \\
\hline pHM2::rhpR-pro-144TG-luc & Derived from *, with $-144 \mathrm{~T}$ replaced by $\mathrm{G}$ & This study \\
\hline pHM2::rhpR-pro-143CA-luc & Derived from *, with $-143 \mathrm{C}$ replaced by A & This study \\
\hline pHM2::rhpR-pro-136GT-luc & Derived from *, with $-136 \mathrm{G}$ replaced by $\mathrm{T}$ & This study \\
\hline pHM2::rhpR-pro-135AC-luc & Derived from $*$, with $-135 \mathrm{~A}$ replaced by $\mathrm{C}$ & This study \\
\hline pHM2::rhpR-pro-134TG-luc & Derived from *, with $-134 \mathrm{~T}$ replaced by G & This study \\
\hline pHM2::rhpR-pro-133AC-luc & Derived from *, with $-133 \mathrm{~A}$ replaced by $\mathrm{C}$ & This study \\
\hline pHM2::rhpR-pro-132CA-luc & Derived from *, with $-132 \mathrm{C}$ replaced by A & This study \\
\hline pHM2::rhpR-pro-141D-luc & Derived from $*$, with $-141 \mathrm{G}$ deleted & This study \\
\hline pHM2::rhpR-pro-141-142D-luc & Derived from $*$, with $-141 \mathrm{G}$ and $-142 \mathrm{C}$ deleted & This study \\
\hline pHM2::rhpR-pro-14111-luc & Derived from *, with 1 adenine inserted between $-141 \mathrm{G}$ and $-142 \mathrm{C}$ & This study \\
\hline pHM2::rhpR-pro-14114-luc & Derived from $*$, with 4 adenines inserted between $-141 \mathrm{G}$ and $-142 \mathrm{C}$ & This study \\
\hline pHM2::PSPTO2767-pro-238-luc & PSPTO2767-luc (-238 from ATG, containing inverted repeat [IR]) reporter in pHM2 & This study \\
\hline pHM2::PSPTO2767-pro-222-luc & PSPTO2767-luc (-222 from ATG, without IR) reporter in pHM2 & This study \\
\hline pHM2::PSPTO2036-pro-109-luc & PSPTO2036-luc (-109 from ATG, containing IR) reporter in pHM2 & This study \\
\hline pHM2::PSPTO2036-pro-93-luc & PSPTO2036-luc (-93 from ATG, without IR) reporter in pHM2 & This study \\
\hline pGST::rhpR & $r h p R$ in pGEX-KG plasmid & This study \\
\hline pGST::rhpR-D70A & Derived from pGST- $r h p R$, with Asp70 replaced by Ala & This study \\
\hline
\end{tabular}


tivity, whereas the 132-bp promoter without the IR element exhibited low activity in the $\operatorname{rhpS} S^{-}$mutant (Fig. 4A). These results indicated that the IR element mediates the RhpR-dependent induction of the $r h p R$ promoter.

The IR sequences of the IR element are perfectly conserved in the promoters of $r h p R$ orthologs in $P$. syringae pv. tomato, $P$. syringae pv. syringae, and $P$. syringae pv. phaseolicola (Fig. 4B). However, the 6-bp spacers are variable in sequence (Fig. 4B). All the IR elements are 132 bp upstream of their corresponding $r h p R$ open reading frames (ORF). Additional elements similar to this IR element were not found in the $r h p R$ promoters.

\section{Determination of the $r h p R$ transcriptional start site.}

To define the position of the IR element in $r h p R$ promoter, a $5^{\prime}$ rapid amplification of cDNA ends (RACE) reaction was performed with RNA samples prepared from WT DC3000 and the
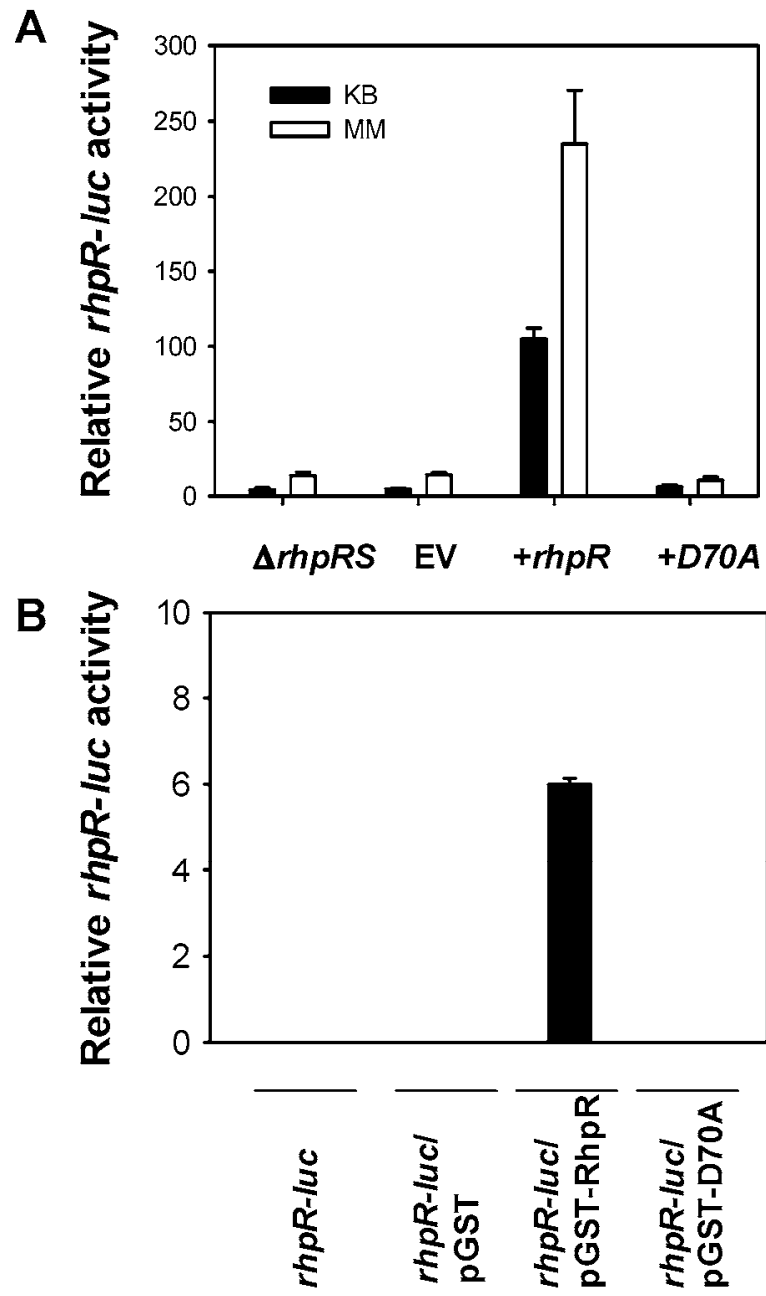

Fig. 2. RhpR requires the phosphorylation site for the induction of $r h p R$ promoter. A, Hemagglutinin (HA)-tagged $r h p R$ and $r h p R(D 70 A)$ genes under the constitutive $\mathrm{pNm}$ promoter in the pML122 plasmid were expressed in the $\triangle r h p R S$ mutant carrying the pHM2::rhpR $R_{540^{-}}$luc reporter. The $\Delta r h p R S$ strain carrying no plasmid and the $\triangle r h p R S$ strain carrying the empty pML122 vector (EV) were included as control. Bacteria were cultured in Kings' B (KB) medium and then induced in minimal medium (MM). Luciferase activities were measured at $0 \mathrm{~h}(\mathrm{~KB})$ and $6 \mathrm{~h}(\mathrm{MM})$ after incubation in $\mathrm{MM}$ and normalized to bacterial numbers. B, $\operatorname{rhpR}$ and $\operatorname{rhpR}(D 70 A)$ genes were cloned into the pGST plasmid and introduced into the Escherichia coli BL21 strain carrying the pHM2::rhpR $R_{540}-l u c$ reporter. Bacterial strains were cultured in Luria-Bertani medium and induced with isopropyl-thio-galactopyranoside (IPTG) for the production of GST-RhpR and GST-RhpR(D70A) fusion proteins. Luciferase activities were measured $1 \mathrm{~h}$ after IPTG induction and normalized to bacterial numbers. Each data point represents the average of three measurements. Error bars indicate standard deviations. $r h p S^{-}$mutant to determine the transcriptional start site of $r h p R$. Four clones containing the PCR products derived from each $5^{\prime}$ RACE reaction were sequenced. All the clones derived from WT DC3000 showed that the 5' end of rhpR RNA starts at $\mathrm{T}_{165}$, which is $165 \mathrm{bp}$ upstream of the $\operatorname{rhpR}$ ORF (Fig. 4C). All the clones derived from the $r h p S^{-}$mutant showed that the $r h p R$ RNA starts at $\mathrm{G}_{87}$, which is $87 \mathrm{bp}$ upstream of the $r h p R$ ORF (Fig. 4C). $\mathrm{G}_{87}$ is conserved in P. syringae pv. tomato and $P$. syringae pv. syringae but not in $P$. syringae pv. phaseolicola, whereas $\mathrm{T}_{165}$ is not conserved in the three sequenced $P$. syringae genomes (Buell et al. 2003; Feil et al. 2005; Joardar et al. 2005). A putative sigma 70 promoter element was predicted at the -10 and -35 regions upstream of $\mathrm{T}_{165}$, and a sigma 54 promoter element of poor homology to the consensus sequence was predicted between the IR element and $\mathrm{G}_{87}$ (Fig. 4C). This sigma 54 element is moderately conserved in the sequenced $P$. syringae genomes (Buell et al. 2003; Feil et al. 2005; Joardar et al. 2005). Although the promoter reporter assay suggested a transcriptional start site in the region between -40 and $-80 \mathrm{bp}$, a transcript starting at this region was not identified by the RACE analysis. It is common that TCS have more than one transcriptional start site controlled by different promoters (Bijlsma and Groisman 2003). For example, three transcriptional start sites have been reported for the E. coli TCS locus qseBC (Clarke and Sperandio 2005).

\section{Mutagenesis analysis of the IR element.}

To determine whether the sequence of the IR element is important to the $r h p R$ promoter activity, mutations ( $\mathrm{G}$ to $\mathrm{T}, \mathrm{T}$ to $\mathrm{G}, \mathrm{A}$ to $\mathrm{C}$, and $\mathrm{C}$ to $\mathrm{A}$ ) were generated to each nucleotide of the IR modules in the pHM2::rhpR $R_{147}-l u c$ reporter plasmid, and the resulting mutant plasmids were introduced into the $r h p S^{-}$mutant. Each mutation reduced the promoter activity but not in a uniform fashion (Fig. 4D). Generally, mutants of the upstream 5-bp module ( -147 to -143$)$ showed better promoter activity than mutants of the downstream 5-bp IR module $(-136$ to -132 ), indicating that the downstream IR module is more important in regulating the promoter activity.

To determine whether the spacer length between the two IR modules is critical for promoter activity, four mutants were generated to the spacer in the pHM2:: rhpR $R_{147}-l u c$ plasmid: one mutant carries a deletion of 1 bp $\left(-\mathrm{C}_{142}\right)$, one mutant carries a deletion of 2 bp $\left(-\mathrm{C}_{142}\right.$ and $\left.-\mathrm{G}_{141}\right)$, one mutant carries insertion of one adenine between $-\mathrm{C}_{142}$ and $-\mathrm{G}_{141}$, and one mutant carries insertion of four adenines between $-\mathrm{C}_{142}$ and $-\mathrm{G}_{141}$ (Fig. 4D). A promoter activity assay showed that any change of the spacer length inactivated the promoter (Fig. 4D), indicating that the 6-bp length of the spacer is required for the activity of the IR element.

\section{Genome-wide search of the genes regulated by the putative IR elements.}

The identification of an RhpR-regulated IR element enabled us to search for putative RhpR-regulated promoters in P. syringae. The DC3000 genome was searched for the perfect IR sequence, GTATC-N ${ }_{6}$-GATAC, which uncovered only the $r h p R$ promoter. Because mutant IR elements with one nucleotide substitution exhibited partial activities, we also searched the DC3000 genome for putative IR sequences carrying one variable nucleotide in the repeat modules (NTATC- $\mathrm{N}_{6}$-GATAC, GNATC- $\mathrm{N}_{6}$-GATAC, GTNTC-N ${ }_{6}$-GATAC, GTANC-N ${ }_{6}-$ GATAC, GTATN-N 6 -GATAC, GTATC-N 6 -NATAC, GTATC$\mathrm{N}_{6}$-GNTAC, GTATC-N 6 -GANAC, GTATC-N 6 -GATNC, and GTATC-N 6 -GATAN). The search produced 44 hits (Table 3 ).

Eighteen genes downstream of these putative IR elements were analyzed using RNA blotting for their expression in WT DC3000, the $r h p S^{-}$mutant, and the $\Delta r h p R S$ mutant (Fig. 5). 
These genes were selected because, according to a microarray analysis, they displayed a differential expression in WT DC3000 and the rhpS $S^{-}$mutant cultured in MM (L. Lan and X. Tang, unpublished data). The PSPTO2036 gene promoter contains a putative IR element (GTATC-N 6 -CTTAC) with two variable nucleotides (underlined) in the downstream IR module. This gene was also selected for RNA blot analysis, because it was expressed at a much higher level in the $\operatorname{rhpS} S^{-}$mutant than in WT DC3000 according to the microarray analysis (unpublished data). RNA blot analysis indicated that five genes, including PSPTO2767, PSPTO2036, PSPTO3477, PSPTO3574, and PSPTO3600, displayed the same expression pattern as that of the $r h p R$ promoter (e.g., more transcripts in the $\operatorname{rhp} S^{-}$mutant than in WT DC3000 and the $\Delta r h p R S$ mutant) (Fig. 5). These genes are probably induced by RhpR. An extra band of larger size was visible in the Northern blot probed by the full-length PSPTO2036 cDNA. This band likely resulted from nonspecific hybridization because it was visible when the $3^{\prime}$ fragment of the PSPTO2036 cDNA was used as probes but not visible when the $5^{\prime}$ fragment was used as probes (data not shown). The $3^{\prime}$ fragment of the PSPTO2036 cDNA is highly rich in GC (approximately 80\%). Two genes, PSPTO0536 and PSPTO0897, were expressed at lower levels in the $r h p S^{-}$mutant than in WT DC3000 and the $\Delta r h p R S$ mutant (Fig. 5). These genes may be suppressed by RhpR. The putative functions of these genes are summarized in Table 3. The remaining genes displayed an expression pattern independent of RhpR.

\section{Function of the putative IR elements in PSPTO2767 and PSPTO2036 promoters.}

The PSPTO2767 and PSPTO2036 transcripts were strongly induced in the $r h p S^{-}$mutant. The putative IR elements were ana-

Table 2. Primers

\begin{tabular}{|c|c|c|c|}
\hline Primer & Sequence & Primer & Sequence \\
\hline DC-rhpR-F & AACATATGATGCAAGCACTTCCCGAC & PSPTO3574-R & TTAAAACGTGACGCTGGCGC \\
\hline DC-rhpR-R & AA $\overline{\text { GGATCCACCCAGCTCCCTGGCATC }}$ & PSPTO3660-F & GTGATTCAGTTCCTTTTG \\
\hline DC-rhpR-pro-540F & TTGAATTCAAACGTCCTTGTTCAACG & PSPTO3660-R & TCAGACATAGTCGGTCAC \\
\hline DC-rhpR-pro-300F & TTGAATTCTTTAAGCCAGCCGAAACC & PSPTO0536-F & ATGGCTGGATTAATGATCGA \\
\hline DC-rhpR-pro-170F & TTGAATTCGTGGTTCGTTCACTCATC & PSPTO0536-R & TTATAACGGCGGTTTCGCGG \\
\hline DC-rhpR-pro-147F & TTGAATTCGTATCCGTATCGATACATTC & PSPTO0897-F & ATGTCGTGCAGAATCATAGTG \\
\hline DC-rhpR-pro-132F & TTGAATTCATTCACGACATCCGCCTG & PSPTO0897-R & TTACCTGATAGCCAGGCTGG \\
\hline DC-rhpR-pro- $120 \mathrm{~F}$ & TTGAATTCCCGCCTGACATCGGCCAG & PSPTO0406-F & ATGAAAAGCCAAACCGATGC \\
\hline DC-rhpR-pro-80F & TTGAATTCACGGCGAAGTAGCATGAG & PSPTO0406-R & ACGCGTCAGTTTTTCGATCAG \\
\hline DC-rhpR-pro-40F & TTGAATTCCGGCCAGACAACGGCGGC & PSPTO0898-F & ATGAAAGACCGGAAAAACGC \\
\hline DC-rhpR-pro-R & TTGGATCC CATAGTGCGTCTGTCGCC & PSPTO0898-R & TTTCGCGAACCGACAGCAGGTTCA \\
\hline DC-rhpR-proF147GT & TTGAATTCTTATCCGTATCGATACATTC & PSPTO1065-F & ATGACCACCCCGCCCGCG \\
\hline DC-rhpR-proF146TG & TTGAATTCGGATCCGTATCGATACATTC & PSPTO1065-R & TATCGGAACAGGGTGTC \\
\hline DC-rhpR-proF145AC & TTGAATTCGTCTCCGTATCGATACATTC & PSPTO1066-F & ATGAACAGTCTTTTGTCACC \\
\hline DC-rhpR-proF144TG & TTGAATTCGTAGCCGTATCGATACATTC & PSPTO1066-R & CGCCGTATTGTCATTCAGCG \\
\hline DC-rhpR-proF143CA & TTGAATTCGTATACGTATCGATACATTC & PSPTO1543-F & GTGCGTAAGTTGACTCAATTG \\
\hline DC-rhpR-proF136GT & TTGAATTCGTATCCGTATCTATACATTC & PSPTO1543-R & TTACTTCAGCTGGTTCATGC \\
\hline DC-rhpR-proF135AC & TTGAATTCGTATCCGTATCGCTACATTC & PSPTO1903-F & ATGACTCAGCTAGAAAAAGC \\
\hline DC-rhpR-proF134TG & TTGAATTCGTATCCGTATCGAGACATTC & PSPTO1903-R & CTACTTCCAGTTCGAGGCCTTC \\
\hline DC-rhpR-proF133AC & TTGAATTCGTATCCGTATCGATCCATTC & PSPTO2749-F & ATGAAGCTTTTCAGACTA \\
\hline DC-rhpR-proF132CA & TTGAATTCGTATCCGTATCGATAAATTC & PSPTO2749-R & TCACAGTTCCGGCCCCAT \\
\hline DC-rhpR-proF142D & TTGAATTCGTATCGTATCGATACATTC & PSPTO3099-F & ATGACCCAGACACTCAGCC \\
\hline DC-rhpR-proF142-141D & TTGAATTCGTATCTATCGATACATTC & PSPTO3099-R & GGAGGCGCTGGCGATTTTGAC \\
\hline DC-rhpR-proF142I1 & TTGAATTCGTATCACGTATCGATACATTC & PSPTO3659-F & ATGACGTTCAAGGCCCCG \\
\hline DC-rhpR-proF142I4 & TTGAATTCGTATCAAAACGTATCGATACATTC & PSPTO3659-R & TCAGCTCGCTTCCAGAGC \\
\hline DC-rhpR-RTP & 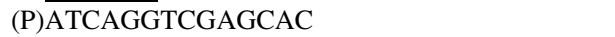 & PSPTO3796-F & ATGAATGGTGAAATGCAGAC \\
\hline DC-rhpR-PE1 & CATAGTGCGTCTGTCGCC & PSPTO3796-R & ААССТCTTCGCCCTCACCCATC \\
\hline DC-rhpR-PE2 & GGTCGATGTGGCATCAAG & PSPTO5198-F & ATGCCAGCAGCCTCCCTC \\
\hline DC-rhpR-S1 & TGACCGACGGTTCGCAGATG & PSPTO5198-R & TCAAAACGGCGCAGTGCC \\
\hline DC-rhpR-S2 & CAGGCACTCACCGATGAAAC & PSPTO5200-F & TTGTGGACCACCGGCGCG \\
\hline rhpR-GST-F & AATGTCTAGAACGCACTATGCAAGCAC & PSPTO5200-R & TTAAAACGCCAACGTCAC \\
\hline rhpR-GST-R & AACTAAGCTTGATCAACCCAGCTCCCTG & PSPTO2767-pro238F & TTGAATTCGTATCAACCTGGGTACAA \\
\hline PSPTO1489-pro101F & GCAGGAACCCCTCTCGTTATC & PSPTO2767-pro222F & TTGAATTCAACTAGTGGCCAAACAAT \\
\hline PSPTO1489-pro21R & GGCAACCTCTCGTAATGAAAA & PSPTO2767-proR & TTGGATCCCATGACTACCCTGTGAGCAC \\
\hline PSPTO2223-pro188F & TTTTAAGGCGTAAGCGTCGT & PSPTO2036-pro109F & TTEAATTCGTATCGCGCCGCTTACAG \\
\hline PSPTO2223-pro12+8R & GCTTGCATAGTGCGTCTGTC & PSPTO2036-pro93F & TTGAATTCAGGTTGGCGACCAGATCG \\
\hline PSPTO2036-pro232F & CTGTGTTTCCGTGTGGGTTT & PSPTO2036-proR & TTGGATCCCATCCGGATTCACTCTCTC \\
\hline PSPTO2036-pro97R & ATCTGGTCGCCAACCTGTAA & PSPTO5200-pro175F & GGTCTGCGCCTTATTCAAAC \\
\hline PSPTO2767-pro400F & TCATTCCGGGCTATCTGAAG & PSPTO5200-pro52R & TGATCCTGTCGTCACCTGAG \\
\hline PSPTO2767-pro184R & AATAGCGGGGCTAAGTCGAT & PSPTO3659-pro217F & GCCAATTGGGTCAATTTGTT \\
\hline PSPTO3477-pro283F & CGCTGCTACGACACTGATGT & PSPTO3659-pro110R & GCACGAAATACGCAAAACCT \\
\hline PSPTO3477-pro305R & GCAGGCAGTAGCACAGGTTA & DChrpR-pro166F & AGCCTGAGTCTATCGGTAGGG \\
\hline PSPTO0536-pro147F & TCAGACATTGGTCTGGTTGC & DChrpR-pro36R & GGGTGGCAAGCGGAGTATTA \\
\hline PSPTO0536-pro22R & GTGGCTCAAGTCCGTGTTTG & DChrpL-pro178F & AGCTGACCGATGTTTTTGTG \\
\hline PSPTO0897-pro114F & TCTTTCCCGAACGTCGATAC & DChrpL-pro58R & CGATAACCATGCCAGCTTAAA \\
\hline PSPTO0897-pro21R & CCTCTGCTTCCGGTATTTTA & DCRpoN-pro110F & ACTCTAGGCAAAGGCACAGG \\
\hline PSPTO0898-pro179F & GTATCGACGTTCGGGAAAGA & DCRpoN-pro20R & GGCAGGGGCTAAACACCTTA \\
\hline PSPTO0898-pro54R & TTAAGGCTCCAGGCTCATTG & PSPTO2036-F & ATGTTGAGTCGAGTAGCAAG \\
\hline PSPTO0406-pro240F & CCTCTCGAGAAGCTTGAACC & PSPTO2036-R & TTAGCGCTCGCCGCCACCC \\
\hline PSPTO0406-pro111R & GAGACCACAGTGGCTTAGTGC & PSPTO2767-F & ATGACTAGTCCATCTATCATTG \\
\hline PSPTO1066-pro167F & ACTTGCTACGAAAGCGATCC & PSPTO2767-R & TTAATGACCGATTACTGCGTC \\
\hline PSPTO1066-pro58R & AAACAGAGGCGATGCATTTT & PSPTO3477-F & TTGGTCAAGCAGTTCCAATC \\
\hline PSPTO5198-pro127F & CGACAAAGTATGCGGACGTA & PSPTO3477-R & TTACTCGCTGGCCTTGAAGC \\
\hline PSPTO5198-pro27R & TCCAACTCCAGAACAGTGTGA & PSPTO3574-F & TTGAGCATCTCCTCCCAACG \\
\hline
\end{tabular}


lyzed for their roles in regulating PSPTO2767 and PSPTO2036 promoters. PSPTO2767 and PSPTO2036 promoters with the IR element (238 bp upstream of PSPTO2767 ORF and 109 bp upstream of PSPTO2036 ORF) and without the IR element (222 bp upstream of the PSPTO2767 ORF and 93 bp upstream of the PSPTO2036 ORF) were fused with the $l u c$ reporter gene and assayed in WT DC3000, the rhpS $S^{-}$mutant, and the $\Delta r h p R S$ mutant (Fig. 6A and B). Both promoters with the IR element showed higher LUC activity in the $\operatorname{rhpS} S^{-}$mutant than in WT DC3000 and the $\Delta r h p R S$ mutant. However, both promoters without the IR element showed low activities in all three strains. These results indicated that the putative IR elements in promoters of PSPTO2767 and PSPTO2036 conferred the RhpR-dependent induction of these genes in the $\operatorname{rhpS} \mathrm{S}^{-}$mutant.

PSPTO2767 encodes a lipopolysaccharide core biosynthesis domain protein. The ortholog of PSPTO2767 in P. syringae pv. syringae, Psy_2496, has two putative IR elements: one is identical to the PSPTO2767 IR element in the repeat modules and the other is identical to the IR element in the promoter of PSPPH_2653, the ortholog of PSPTO2767 in P. syringae pv. phaseolicola (Fig. 6C). There is no additional IR element in the DC3000 genome identical to the IR element of PSPTO2767 in the repeat modules.

PSPTO2036 encodes a putative small lipoprotein, and its orthologs in the $P$. syringae pv. phaseolicola and $P$. syringae pv. syringae genomes were not annotated (Feil et al. 2005; Joardar et al. 2005). Tblastn search identified a small ORF (named PSPPH1805^6) between PSPPH1805 and PSPPH1806 in the $P$. syringae pv. phaseolicola genome and a small ORF (named Psy1846^7) between Psy_1846 and Psy_1847 in the $P$. syringae pv. syringae genome (Buell et al. 2003; Feil et al. 2005; Joardar et al. 2005). Proteins encoded by these small genes are identical in the N-terminal signal peptide but variable in the $\mathrm{C}$-terminal portion following the lipid modification site (data not shown). The IR elements of these genes are identical in the repeat modules (Fig. 6C). No additional IR element was found in the DC3000 genome identical to the IR element of PSPTO2036 in the repeat modules. Northern blot analysis indicated that PSPPH $1805^{\wedge} 6$ was expressed at a higher level in the $\operatorname{rhpS} S^{-}$mutant than in the WT P. syringae pv. phaseolicola strain (data not shown). The promoters of PSPTO2767 and PSPTO2036 and their orthologous genes in $P$. syringae pv. syringae and $P$. syringae pv. phaseolicola all carry a putative sigma 54 element downstream of the IR element (data not shown).

\section{RhpR binds the promoters containing a putative IR element.}

ChIP and qRT-PCR assays were performed to test whether RhpR binds the promoters carrying a putative IR element. The promoters of 11 genes were tested. Three of these genes (PSPTO2767, PSPTO2036, and PSPTO3477) displayed an RhpR-dependent upregulation, two genes (PSPTO0536 and PSPTO0897) displayed an RhpR-dependent downregulation, and six genes (PSPTO0898, PSPTO0406, PSPTO1066, PSPTO5198, PSPTO5200, and PSPTO3659) displayed an RhpR-independent expression (Fig. 5). Except for the promoter DNA of PSPTO3659, the remaining 10 promoter DNAs all exhibited a clear RhpR-dependent enrichment in ChIP assay (Fig. 3), even though some of the corresponding genes did not show an RhpR-dependent regulation in Northern blot analysis (Fig. 5).

Because RhpR represses the induction of genes in the T3 gene regulatory cascade in MM, we also tested whether RhpR binds the promoters of the known T3 regulatory genes, including $h r p R$, $h r p L$, and rpoN (Xiao and Hutcheson 1994; Xiao et al. 1994; Hendrickson et al. 2000). The promoters of these genes do not contain a putative IR element (Buell et al. 2003). The promoter DNA of these genes did not exhibit an RhpRdependent enrichment in ChIP assay (Fig. 3), indicating that RhpR does not bind these promoters.

\section{DISCUSSION}

Like many bacterial TCS genes, the rhpRS locus is subject to positive autoregulation by RhpR. ChIP and qRT-PCR assays

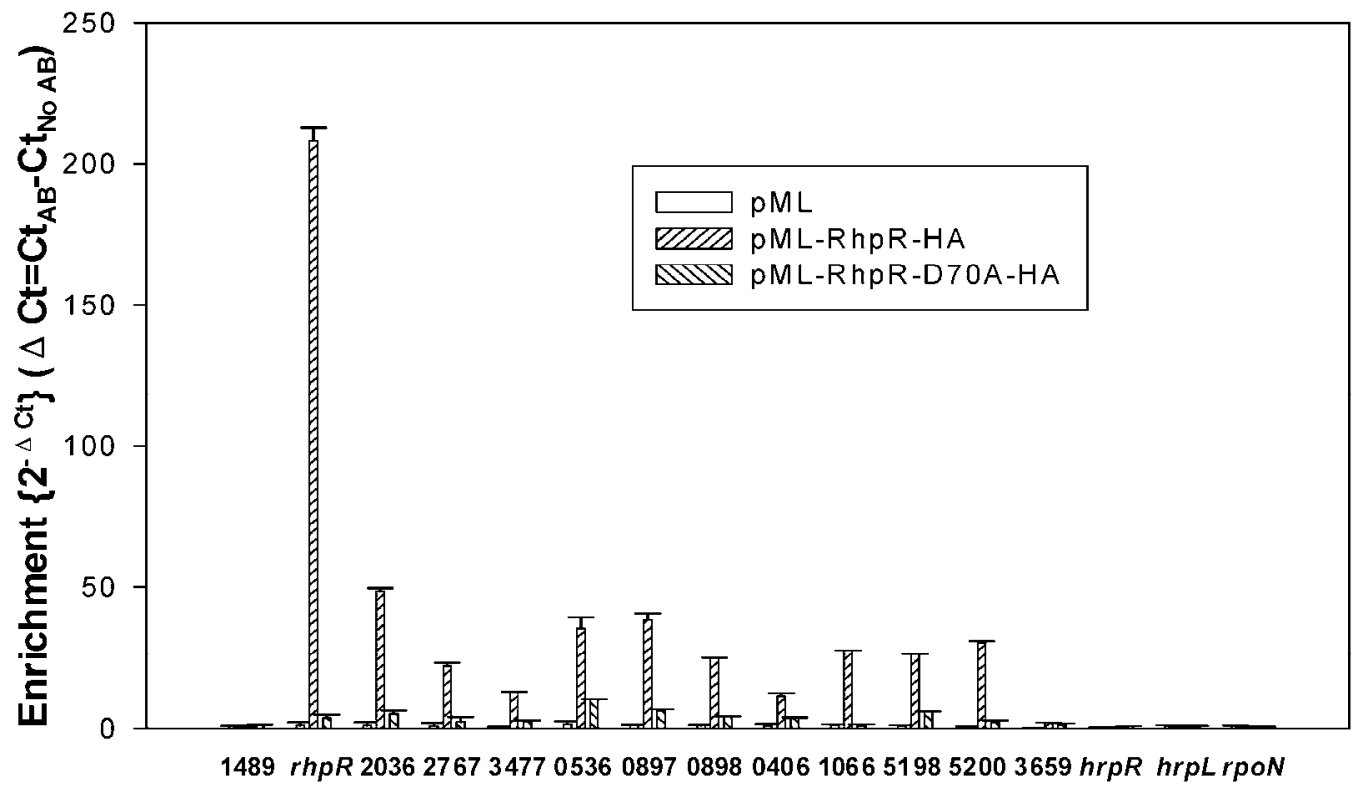

Fig. 3. Chromatin immunoprecipitation (ChIP) quantitative real-time polymerase chain reaction (qRT-PCR) assay of in vivo RhpR binding with promoters. $\triangle r h p R S$ strains containing the pML122 empty vector, pML122::rhpR-HA, and pML122::rhpR(D70A)-HA were used in ChIP assay with and without the antihemagglutinin (HA) antibodies. The strains were grown in King's B medium. Promoter regions of the selected genes in the immunocomplexes were examined by qRT-PCR. Enrichments of promoter DNAs in the immunocomplexes by the anti-HA antibodies (expressed as fold changes) were calculated as $2^{-\Delta \mathrm{Ct}}\left(\Delta \mathrm{Ct}=\mathrm{Ct}_{\mathrm{AB}}-\mathrm{Ct}_{\mathrm{No}} \mathrm{AB}\right)$, where $\mathrm{Ct}=$ threshold cycle and $\mathrm{AB}=$ antibodies. Results are from three independent experiments. Error bars indicate standard deviations. 
indicated that RhpR directly binds to the $r h p R$ promoter. RhpR regulates the $\operatorname{rhpR}$ promoter in a phosphorylation-dependent manner. Mutation of the putative phosphorylation site in RhpR protein (D70A) almost abolished its regulatory activity and association with the rhpR promoter. Based on these results, we propose that phosphorylation of RhpR facilitates its interaction with the $r h p R$ promoter. The $r h p R_{540}$-luc reporter gene dis- played low activity in the $\triangle r h p R S$ mutant in both MM and KB, indicating an RhpR-independent basal expression of the $\operatorname{rhpRS}$ locus. Results from our previous study as well as this study both suggested that, in KB medium, MM, and the plants, RhpS serves as a phosphatase to retain RhpR in an unphosphorylated state (Xiao et al. 2007). Similar interactions between a sensor kinase and the cognate response regulator have been reported

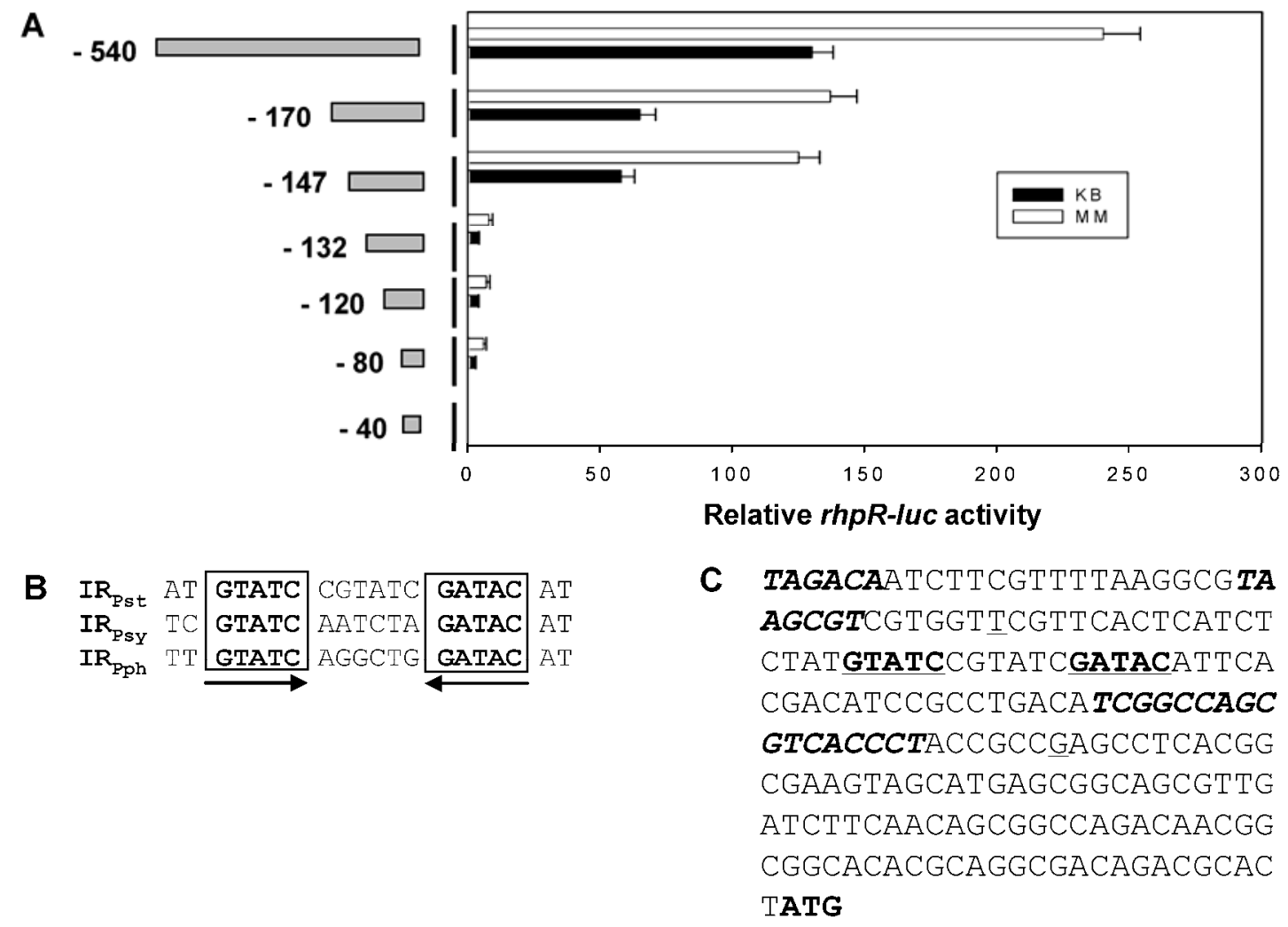

D

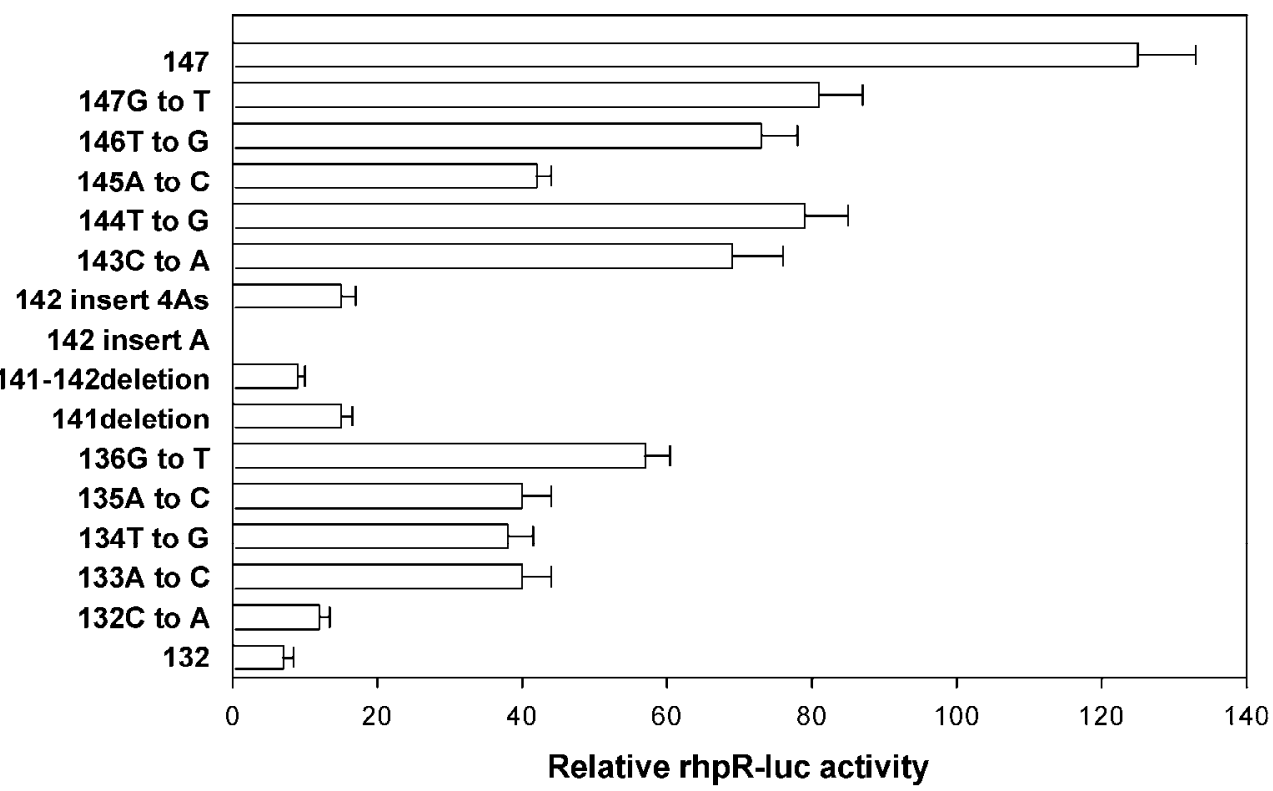

Fig. 4. Identification and mutagenesis analysis of the inverted repeat (IR) element in the $r h p R$ promoter. A, $r h p R_{540}$ promoters of 540, 170, 147, 132, 120, 80, and $40 \mathrm{bp}$ were cloned into the pHM2-luc plasmid. Resulting constructs were introduced into the $\operatorname{rhpS} S^{-}$mutant. Bacteria were cultured in King's B medium and then incubated in minimal medium for $6 \mathrm{~h}$ before the measurement of luciferase activities. Gray bars on the right indicate the length of promoter deletions. Luciferase activities were normalized to bacterial cell numbers. B, Sequence features of the $r h p R$ promoter. Translational start codon ATG of $r h p R$ is bold. The transcriptional start sites G87 and T165 are underlined. The IR element is bold and underlined. The putative sigma 70 and sigma 54 sites are bold italic. C, Alignment of the IR elements in the rhpR promoters of Pseudomonas syringae pv. tomato DC3000, P. syringae pv. syringae B728a, and P. syringae pv. phaseolicola 1448A strains. The IR modules are in boxes. D, Mutagenesis analysis of the IR element. The length of the promoters and the mutations of the IR modules are indicated on the left of the figure. Mutant promoters were cloned into the pHM2-luc reporter plasmid. Activities of the mutant promoters were assayed as described in A. Luciferase activities were normalized to bacterial cell numbers. Error bars indicate standard deviations. 
for several TCS, including QseBC of E. coli (Kostakioti et al. 2009), CovRS of S. pyogenes (Dalton and Scott 2004), and VanRS of Streptomyces coelicolor (Hutchings et al. 2006). Based on this study and results from other TCS, we speculate that environmental signals stimulate the RhpS kinase activity which, in turn, phosphorylates RhpR, and the phosphorylated $\mathrm{RhpR}$ (P-RhpR) binds to the $\operatorname{rhpR}$ promoter and activates the expression of rhpRS, leading to rapid accumulation of RhpS and RhpR proteins and quick response to the signal.

Deletion analysis revealed a perfect IR element in the $\operatorname{rhpR}$ promoter that confers the RhpR-dependent gene regulation. Although we were unable to demonstrate the direct interaction of RhpR protein with the IR element due to the failure to obtain purified RhpR protein, based on the requirement of the IR element for the RhpR-dependent induction of $r h p R$ promoter, we propose that $\mathrm{P}-\mathrm{RhpR}$ protein forms a homodimer on the IR element. Point mutations of the repeat modules reduced but did not abolish the $\operatorname{rhpR}$ promoter ac- tivity, suggesting that P-RhpR can dimerize on an imperfect IR element. However, alteration of the spacer length between the repeats completely abolished the promoter activity, suggesting that the space between the two repeat modules is crucial either for dimerization of P-RhpR on the IR element or the engagement of P-RhpR protein with the RNA polymerase complex. This result also suggested that a single repeat module sequence in the promoter is unlikely to have an RhpR-dependent regulatory activity.

Identification of the IR element enabled computational search of the RhpR-regulated genes in the DC3000 genome. The IR element in the $r h p R$ promoter is the only perfect IR element in DC3000. Because mutagenesis analysis indicated that point mutations of the repeat modules only reduced the IR activity, our initial search focused on putative IR elements with one nucleotide mismatch. In all, 44 putative IR elements of this type were identified. Many of these 44 putative IR elements are in the promoters of conserved genes in the three sequenced $P$. syringae

Table 3. Genes containing a putative inverted repeat (IR) element in their promoters

\begin{tabular}{|c|c|c|c|}
\hline Gene $^{a}$ & Function & Position & IR sequence \\
\hline \multicolumn{4}{|l|}{ Group I } \\
\hline PSPTO2223* & RhpR & -147 to -132 & GTATCCGTATCGATAC \\
\hline PSPTO2036* & Lipoprotein, putative & -109 to -94 & GTATCGCGCCGCTTAC \\
\hline PSPTO2767* & LPS core biosynthesis domain protein & -238 to -222 & GTATCAACCTGGGTAC \\
\hline PSPTO3477* & Hypothetical & -290 to -275 & GTATCGCCGCTGCTAC \\
\hline PSPTO3574 & TonB-dependent siderophore receptor, putative & -103 to -88 & GTTTCAAGACTGATAC \\
\hline PSPTO3660 & Xanthine dehydrogenase & -231 to -216 & GTATCGCAACCGATGC \\
\hline \multicolumn{4}{|l|}{ Group II } \\
\hline PSPTO0536* & Sensory box/GGDEF domain/EAL domain protein & -99 to -84 & GTATCACCCCGGACAC \\
\hline PSPTO0897* & DNA-binding response regulator, LuxR family & -68 to -53 & GTAACACAGACGATAC \\
\hline \multicolumn{4}{|l|}{ Group III } \\
\hline PSPTO0406* & Sensory box/GGDEF domain/EAL domain protein & -163 to -148 & GTATCCGACCAGTTAC \\
\hline PSPTO0898* & Sensor histidine kinase/response regulator & -221 to -206 & GTATCGTCTGTGTTAC \\
\hline PSPTO1065 & DnaJ domain protein & -248 to -223 & GTTTCAATGGCGATAC \\
\hline PSPTO1066* & Methyl-accepting chemotaxis protein & -111 to -96 & GTATCGCCATTGAAAC \\
\hline PSPTO 1543 & Outer membrane protein $\mathrm{OmpH}$ & -272 to -257 & GTAACGTATTTGATAC \\
\hline PSPTO1903 & Hypothetical & -109 to -94 & GTATACGAGGCGATAC \\
\hline PSPTO2749 & Hypothetical & -217 to -202 & GTATCGTCGTTGACAC \\
\hline PSPTO3099 & MexE, multidrug efflux membrane fusion protein & -288 to -273 & GTATATTTCGGGATAC \\
\hline PSPTO3659* & Transcriptional regulator, GntR family & -59 to -44 & GCATCGGTTGCGATAC \\
\hline PSPTO3796 & GGDEF domain protein & -306 to -291 & GTATCATTCGTGATTC \\
\hline PSPTO5198* & Dioxygenase, TauD/TfdA family & -153 to -138 & GTATCTGCCGTGACAC \\
\hline \multirow[t]{2}{*}{ PSPTO5200(2)* } & Autotransporter, putative & -218 to -203 & GTGTCACGGCAGATAC \\
\hline & $\ldots$ & -105 to -90 & GTGTCGTCCCTGATAC \\
\hline \multicolumn{4}{|l|}{ Group IV } \\
\hline PSPTO0076 & Hypothetical & -326 to -311 & GTTTCATCTGGGATAC \\
\hline PSPTO0095 & Phospholipase D family protein & -71 to -56 & GTATCGTGGGCGAGAC \\
\hline PSPTO0189 & Nitrilase, putative & -154 to -139 & GTATCGAAAAAGATGC \\
\hline PSPTO0769 & $\mathrm{ABC}$ transporter, ATP-binding protein & -78 to -63 & GTATCGCGCCTGAAAC \\
\hline PSPTO1468 & PurT, phosphoribosylglycinamide formyltransferase 2 & -255 to -240 & GCATCCCGCTGGATAC \\
\hline PSPTO1566 & Hypothetical & -36 to -21 & GTCTCACCCTCGATAC \\
\hline PSPTO 1567 & ISPsy6, transposase & -367 to -352 & GTATCGAGGGTGAGAC \\
\hline PSPTO2053 & Hypothetical & -354 to -339 & GTAACGTATCAGATAC \\
\hline PSPTO2055 & SpeE, spermidine synthase & -206 to -191 & GTATCTGATACGTTAC \\
\hline PSPTO2185 & EtfB-2, electron transfer flavoprotein & -281 to -266 & GTAACGGTCAAGATAC \\
\hline PSPTO2224 & Hypothetical & -250 to -235 & GTATCGATACGGATAC \\
\hline PSPTO2292 & Phosphoenolpyruvate synthase & -246 to -231 & GTTTCGGCGGTGATAC \\
\hline PSPTO2794 & Hypothetical & -57 to -42 & GTATAGCCGTCGATAC \\
\hline PSPTO2885 & Transposase_34 & -148 to -133 & GTATCGAGTGCGATAG \\
\hline PSPTO3098 & Methyl-accepting chemotaxis protein & -29 to -14 & GTATCCCGAAATATAC \\
\hline PSPTO3254 & Transcriptional regulator, GntR family & -44 to -29 & GTATCGCAGCCTATAC \\
\hline PSPTO3266 & Phosphate ABC transporter & -232 to -217 & GTATCACCGGCGAAAC \\
\hline PSPTO3478 & Hypothetical & -230 to -215 & GTAGCAGCGGCGATAC \\
\hline PSPTO3797 & Hypothetical & -107 to -92 & GAATCACGAATGATAC \\
\hline PSPTO3903 & Hypothetical & -150 to -135 & TTATCAGCGTAGATAC \\
\hline PSPTO3913 & Hypothetical & -347 to -332 & GTATCGGTGCAGAAAC \\
\hline PSPTO4154 & Hypothetical & -334 to -319 & GTATCAAAACAGATGC \\
\hline PSPTO 4588 & HopS2, type three effector & -226 to -211 & GTATCGCGCTGGATAT \\
\hline PSPTO5482 & Response regulator & -252 to -237 & GTATCTCGAGCGAAAC \\
\hline
\end{tabular}

${ }^{a}$ Group I, upregulated genes in the $r h p S^{-}$mutant; group II, downregulated genes in the $r h p S^{-}$mutant; group III, unchanged genes in the rhpS ${ }^{-}$mutant; and group

IV, untested genes. Genes in groups I to III were tested by RNA blotting. Genes indicated by * were tested by chromatin immunoprecipitation analysis. 
genomes (Buell et al. 2003; Feil et al. 2005; Joardar et al. 2005). Some of these IR elements may be functional, because the RhpR proteins in P. syringae strains are $>98 \%$ identical (Xiao et al. 2007), and they are likely to regulate conserved functions.
Further characterization of these putative IR elements was guided by the data from a microarray analysis that was designed to compare gene expression in WT DC3000 and the $r h p S^{-}$mutant cultured in MM. Nineteen genes (including one

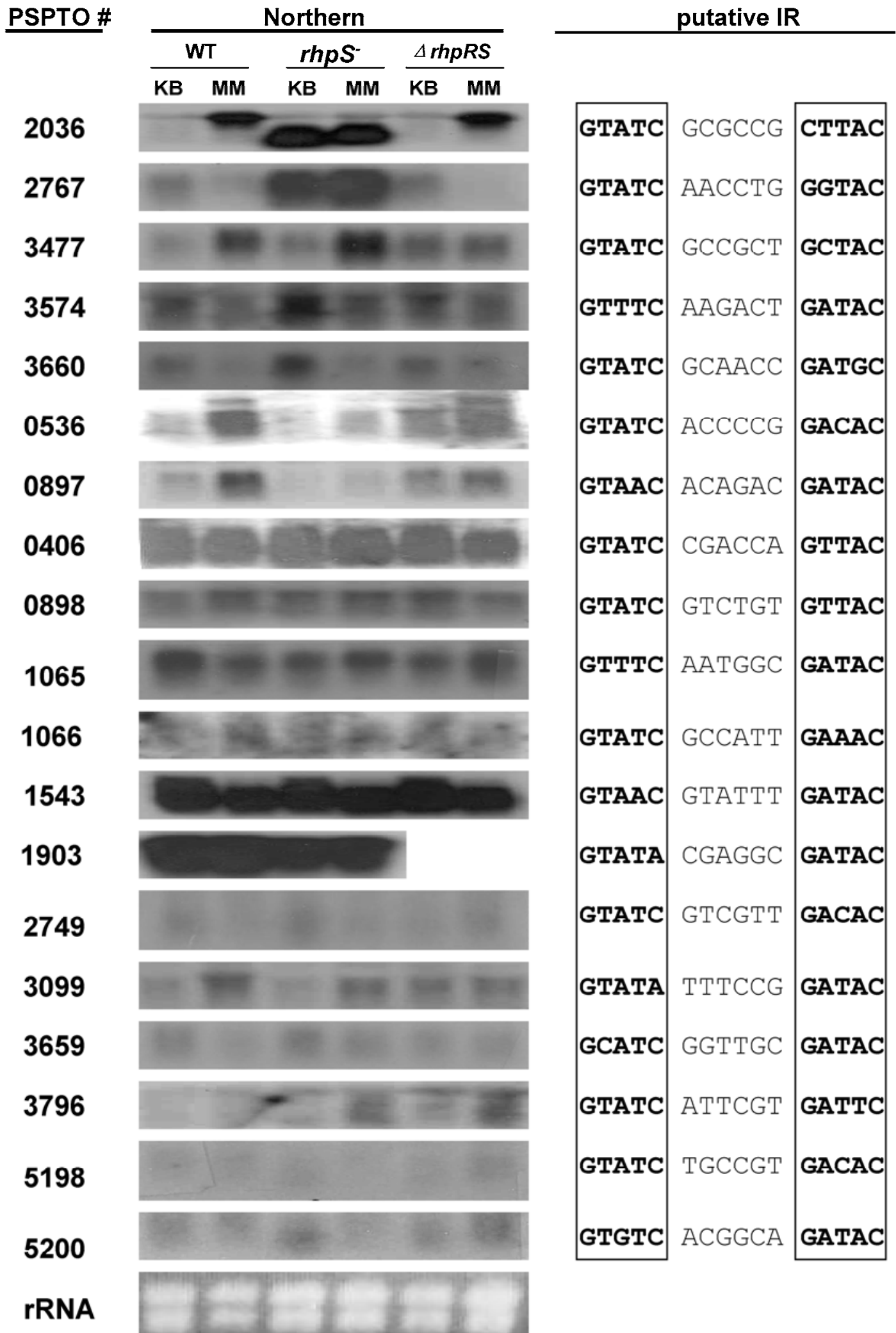

Fig. 5. Northern blot analysis of genes carrying a putative inverted repeat (IR) element in the promoter. Wild-type DC3000 (WT), the rhpS mutant, and the $\triangle r h p R S$ mutant were grown in King's B medium and then incubated in minimal medium for $6 \mathrm{~h}$ before RNA extraction. Total RNA (10 $\mu \mathrm{g} / \mathrm{sample})$ was analyzed by RNA blotting with radio-labeled probes derived from the coding regions of the corresponding genes. The ethidium bromide-stained RNA gel indicates the loading of RNA samples. Sequences of the putative IR elements in the gene promoters are shown in the right column. 
gene with two nucleotide mismatches) that displayed a differential expression in the microarray analysis were assayed for their expression in WT DC3000, the $\operatorname{rhpS} S^{-}$mutant, and the $\Delta r h p R S$ mutant using RNA blot analysis. This assay identified five genes that showed an RhpR-dependent induction and two genes that showed an RhpR-dependent suppression in the rhpS mutant. The IR elements of PSPTO2036 and PSPTO2767 were further assayed for their activities to regulate the corresponding promoters. Both IR elements were
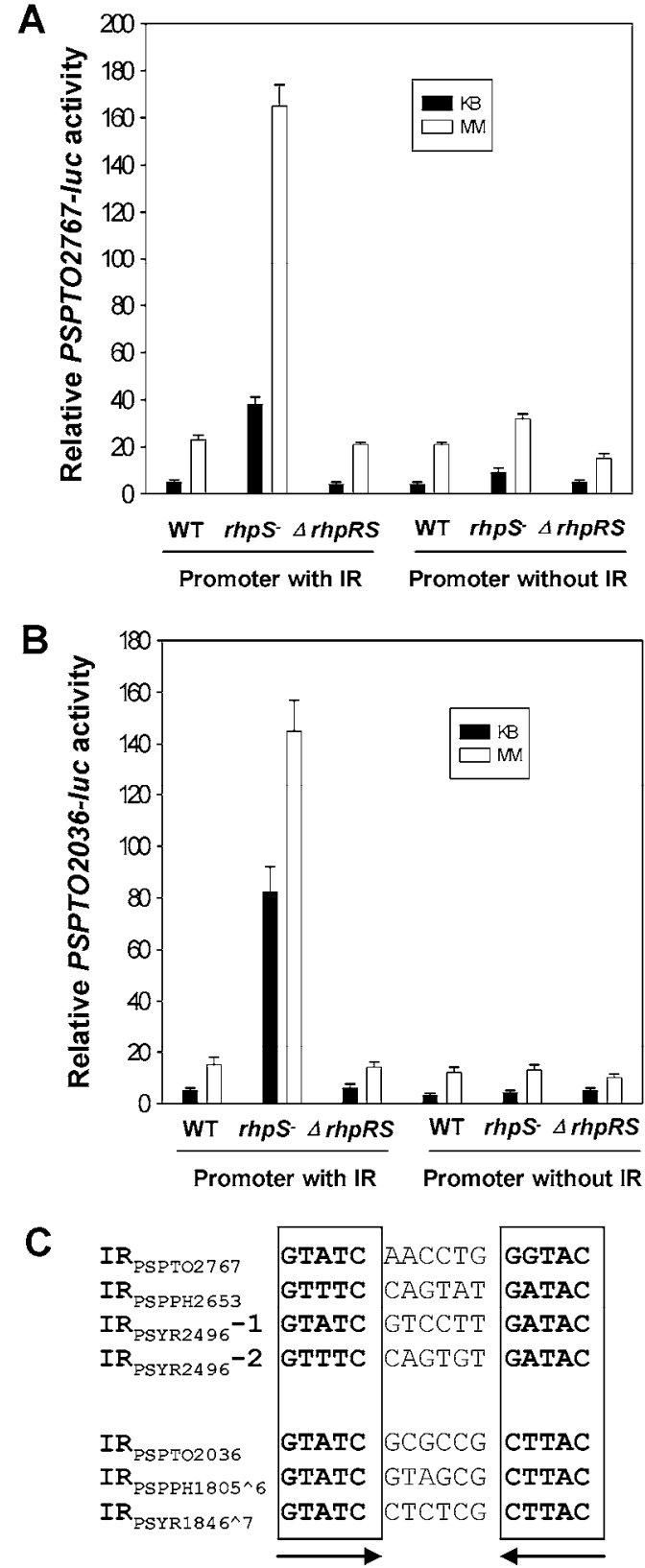

Fig. 6. Characterization of the putative inverted repeat (IR) elements in the promoters of PSPTO2767 and PSPTO2036 genes. A, PSPTO2767 promoters and B, PSPTO2036 promoters with and without the IR element in the pHM2-luc plasmid were introduced into wild-type DC3000 (WT), the rhpS mutant, and the $\Delta r h p R S$ mutant. Bacterial strains were cultured in King's B (KB) medium and then incubated in minimal medium (MM). Luciferase activities were measured at $0 \mathrm{~h}(\mathrm{~KB})$ and $6 \mathrm{~h}(\mathrm{MM})$ after incubation in $\mathrm{MM}$ and normalized to bacterial cell numbers. Error bars indicate standard deviations. C, Sequences of the putative IR elements in the promoters of PSPTO2767 and PSPTO2036 and their orthologs in Pseudomonas syringae pv. syringae $\mathrm{B} 728 \mathrm{a}$ and $P$. syringae $\mathrm{pv}$. phaseolicola 1448A strains. found to be required for the RhpR-dependent induction of the respective promoters. The remaining IR elements have not been characterized for their activities to regulate their corresponding promoters. Further characterization of these IR elements will show whether they, indeed, mediate the RhpR-dependent induction or suppression of their corresponding genes. It should be noted that the IR element in the PSPTO2036 promoter has two nucleotide mismatches in one of the repeat modules but this IR element still confers a strong RhpR-dependent induction, suggesting that a functional IR element can tolerate more than one variable nucleotide in the repeat modules. Thus, future studies of the IR element should test how mutation of two or more nucleotides in the repeat modules affects the activity of the IR element. Such information is crucial for computational identification of the RhpR-regulated genes. All three confirmed IR elements are upstream of a putative sigma-54 binding site, suggesting that $\mathrm{P}-\mathrm{RhpR}$ may interact with the sigma 54 protein to activate the transcription of the corresponding genes. The position of a response-regulator binding site relative to the sigma-factor binding site is crucial to its regulatory activities. In general, response regulators that bind upstream of the sigma-factor binding site positively regulate gene transcription (Bijlsma and Groisman 2003). For PSPTO2767 and PSPTO2036, the upstream position of the IR elements relative to the putative sigma-54 binding site is consistent with the positive role of RhpR. A sigma-factor-regulated promoter element of high homology was not identified in the promoters of PSPTO0536 and PSPTO0897. However, the putative IR motifs and the translation start codons are closely located in both genes. This implies the possibility that the IR motif is downstream of a core promoter and confers the RhpR-dependent negative regulation of the corresponding gene.

PSTTO2767 encodes a putative lipopolysaccharide core biosynthesis domain protein, whereas PSPTO2036 encodes a putative small lipoprotein (Buell et al. 2003). These genes encode conserved functions in the sequenced $P$. syringae genomes (Buell et al. 2003; Feil et al. 2005; Joardar et al. 2005). It is interesting that both genes seem to have a bacterial cell-wallrelated function. The opposite expression patterns of the two cell-wall-related genes and the T3 genes suggested coordination of the T3 gene expression with a cell-wall-related function.

A ChIP assay was used to determine whether RhpR interacts with the promoters carrying a putative IR element. This assay confirmed that RhpR interacts with the three promoters carrying an RhpR-induced IR element (rhpR, PSPTO2767, and PSPTO2036). RhpR also interacts with the promoters of the two genes repressed by RhpR (PSPTO0536 and PSPTO0897). However, it remains to be determined whether RhpR binds the putative IR element in these promoters and whether the binding of RhpR with these promoters mediates the negative regulation of the corresponding genes. Surprisingly, RhpR also interacts with the putative IR promoters that have a putative IR element but did not show an RhpR-dependent regulation. The result is unlikely an artifact of the ChIP assay, because the interaction is specific to the promoters with the IR element, and promoters without the IR element did not show any interaction with the RhpR protein. It is possible that RhpR does, indeed, interact with these promoters, and the regulation of these promoters requires the function of RhpR. However, activation or suppression of these promoters requires additional proteins that were not present in the test growth conditions (i.e., culture in MM and KB medium). Similar results have been reported for the TCS response regulator CovR that interacts with specific sites not found to be regulated by CovR (Churchward et al. 2009).

Although RhpR has been identified as a suppressor of the P. syringae T3 genes, ChIP assays indicated that RhpR does 
not bind directly to the promoters of the T3 regulatory genes, including $h r p R$, $h r p L$, and $r p o N$. These regulatory genes do not have a putative IR element in their promoters. These results suggested that RhpR indirectly regulates the $\mathrm{T} 3$ regulatory cascade consisting of $h r p R S, h r p L$, and the T3 genes. One or more of the genes directly regulated by $\mathrm{RhpR}$ may serve as the link to connect RhpR and the hrpRS-hrpL-T3 gene transcriptional cascade.

\section{MATERIALS AND METHODS}

\section{Bacterial strains and media.}

Bacterial strains used in this study were $P$. syringae pv. tomato DC3000 (Buell et al. 2003) and the rhpS $S^{-}$and $\Delta r h p R S$ mutant strains derived from DC3000 (Xiao et al. 2007). E. coli DH5 $\alpha$ was used for constructing all plasmids. E. coli BL21 was used for testing of RhpR-mediated induction of $r h p R$ promoter. DC3000 and its derivatives were grown at room temperature in KB medium (King et al. 1954) containing appropriate antibiotics. E. coli strains were cultured in Luria-Bertani (LB) medium at $37^{\circ} \mathrm{C}$. Antibiotics for selection of $P$. syringae strains were rifampicin, $25 \mathrm{mg} / \mathrm{liter}$; kanamycin, $10 \mathrm{mg} / \mathrm{liter}$; spectinomycin, $50 \mathrm{mg} / \mathrm{liter}$; tetracycline, $10 \mathrm{mg} / \mathrm{liter}$; and gentamicin, $10 \mathrm{mg} / \mathrm{liter}$. Antibiotics for selection of $E$. coli were ampicillin, $100 \mathrm{mg} /$ liter; kanamycin, $50 \mathrm{mg} /$ liter; spectinomycin, $100 \mathrm{mg} / \mathrm{liter}$; and gentamicin, $20 \mathrm{mg} / \mathrm{liter}$.

\section{Construction of plasmids for promoter analysis.}

All promoter DNA fragments were PCR amplified using the DC3000 genomic DNA as a template. Primers used for PCR amplifications are listed in Table 2. To facilitate cloning, all forward primers were added with an EcoRI site and all reverse primers were added with a BamHI site.

For $r h p R$ promoter deletion analysis, reverse primer rhpRproR was used with one of the forward primers rhpR-pro540F, rhpR-pro170F, rhpR-pro147F, rhpR-pro132F, rhpR-pro120F, rhpR80-pro120F, and rhpR-pro40F to PCR amplify the $r h p R$ promoter fragments of $540,300,170,147,132,120$, and 40 bps upstream of the rhpR ORF.

To create point mutations in the IR element of $r h p R$ promoter, forward PCR primers rhpR-pro147G-TF $\left(-147_{\mathrm{G}}\right.$ to $\left.\mathrm{T}\right)$, rhpR-pro146T-GF (-146 T to $\left._{\mathrm{G}}\right)$, rhpR-pro145A-CF ( $\left.-145_{\mathrm{A} \text { to } \mathrm{C}}\right)$, rhpR-pro144T-GF (-144 To $\left._{\text {G }}\right)$, rhpR-pro143C-AF (-143 to $\left.\mathrm{A}^{\mathrm{A}}\right)$, rhpR-pro136G-TF (-136 G to $)$, rhpR-pro135A-CF $\left(-135_{\mathrm{A} \text { to } \mathrm{C}}\right)$, rhpR-pro134T-GF (-134 $\left.\mathrm{T}_{\mathrm{T}} \mathrm{G}\right)$, rhpR-pro133A-CF (-133 $\left.\mathrm{A}_{\mathrm{A}} \mathrm{C}\right)$, and rhpR-pro132C-AF $\left(-132_{\mathrm{C}}\right.$ to $\left.\mathrm{A}\right)$ were used with the reverse primer rhpR-proR in PCR amplifications.

To insert or delete nucleotides in the spacer of the IR element of $r h p R$ promoter, the forward primers rhpR-pro141I1F (one adenine insertion between $-141_{\mathrm{G}}$ and $-142_{\mathrm{C}}$ ), rhpRpro141I4F (four adenine insertion between $-141_{\mathrm{G}}$ and $-142_{\mathrm{C}}$ ), rhpR-pro141DF (deletion of $-141_{\mathrm{G}}$ ), and RhpR-pro141-142DF (deletion of both $-141_{\mathrm{G}}$ and $-142_{\mathrm{C}}$ ) were used in PCR with the reverse primer rhpR-proR.

A 238-bp fragment (with the putative IR element) and a 222-bp fragment (without the putative IR element) of the PSPTO2767 promoter were PCR amplified using the forward primers PSPTO2767-pro238F and PSPTO2767-pro222F in combination with the reverse primer PSPTO2767-proR.

A 109-bp fragment (with the putative IR element) and a 93bp fragment (without the putative IR element) of the PSPTO2036 promoter were PCR amplified using the forward primers PSPTO2036-pro109F and PSPTO2036-pro93F, respectively, with the reverse primer PSPTO2036-proR.

The PCR products were digested with EcoRI and BamHI, cloned into pBluescript-SK-luc (Deng et al. 2009), sequence verified, and subsequently cloned into the broad host pHM2 plasmid (Xiao et al. 2007). The resulting plasmids (Table 1) were introduced into WT DC3000, the $\operatorname{rhp} S^{-}$mutant, and the $\triangle r h p R S$ mutant by electroporation.

To determine the promoter activities, bacteria containing the promoter reporter genes were grown in $\mathrm{KB}$ medium to an optical density at $600 \mathrm{~nm}$ of $2\left(\mathrm{OD}_{600}=2\right)$, washed twice with $\mathrm{MM}$, resuspended in $\mathrm{MM}$ to $\mathrm{OD}_{600}=0.2$, and cultured at $28^{\circ} \mathrm{C}$ with constant shaking for 0 and $6 \mathrm{~h}$ before the measurement of reporter gene activities. Bacterial culture $(100 \mu \mathrm{l})$ was mixed with $1 \mu \mathrm{l}$ of $1 \mathrm{mM}$ luciferin in a 96-well plate, and the luciferase activities were determined using a cooled CCD camera (Roper Scientific, Trenton, NJ, U.S.A.). The luciferase activities of all measurements were normalized to the bacterial numbers. An $F$ test $(P<0.05)$ was conducted to all quantitative experiments.

\section{Analysis of RhpR-mediated regulation of $r h p R$ promoter in $E$. coli.}

pGST::rhpR and pGST::rhpR(D70A) were constructed to express GST-RhpR and GST-RhpR(D70A) proteins in E. coli BL21. The pML122::rhpR-HA and pML122::rhpR-D70A-HA plasmids (Xiao et al. 2007) were used as template DNA for PCR-amplification of $\operatorname{rhpR}$ and $\operatorname{rhpR}(D 70 A)$, respectively, with rhpR-GST-F (containing an XbaI site) and rhpR-GST-R (containing an HindIII site) as primers (Table 2). The PCR products were digested with $X b a \mathrm{I}$ and HindIII, cloned into pGEX-KG (Guan and Dixon 1991), and sequence verified. pGST::rhpR and pGST::rhpR-D70A were transformed into $E$. coli BL21 containing the reporter plasmid pHM2::rhp $R_{540^{-}}$ luc. To determine the LUC activities, bacterial strains were grown at $37^{\circ} \mathrm{C}$ in $\mathrm{LB}$ medium containing spectinomycin and ampicillin to $\mathrm{OD}_{600}=1$. Isopropyl-thio-galactopyranoside (IPTG) was added into the cultures to a final concentration of $1 \mathrm{mM}$ to induce the GST fusion protein production. At $1 \mathrm{~h}$ after IPTG induction, $100 \mu \mathrm{l}$ of culture was mixed with $1 \mu \mathrm{l}$ of $1 \mathrm{mM}$ luciferin in a 96-well plate, and the luciferase activity was determined using a cooled CCD camera (Roper Scientific).

\section{Determination of the $r h p R$ transcriptional start site.}

5' RACE was performed using the 5'-Full RACE core set (Takara, Shiga, Japan) and total RNAs prepared from WT DC3000 and the rhpS mutant following the manufacturer's instructions. First-strand cDNAs were prepared from $1 \mu \mathrm{g}$ of total RNA with the 5'-phosphorylated reverse-transcription primer, DC-rhpR-RTP (Table 2), and Avian myeloblastosis virus reverse transcriptase. The template RNAs were then digested by RNase $\mathrm{H}$, and the cDNAs were circulated by ligation. The circulated cDNAs was then amplified by nested PCR with two pairs of primers: DC-rhpR-PE1 and DC-rhpR-S1 as the first pair and DC-rhpR-PE2 and DC-rhpR-S2 as the second pair (Table 2). The PCR products were cloned into the pGEM-T vector (Promega Corp., Madison, WI, U.S.A.) and sequenced. Homology of the trapped sequences was searched with the BLASTn program.

\section{RNA isolation and Northern blotting.}

Procedures described by Lan and associates (2006) were used for RNA extraction and Northern blotting. The bacterial strains were grown in KB broth (King et al. 1954) to approximately $\mathrm{OD}_{600}=2$ before being harvested for RNA extraction. For gene expression analysis in MM, the bacteria first were grown in $\mathrm{KB}$ to $\mathrm{OD}_{600}=2$, then centrifuged, washed twice with MM (Huynh et al. 1989), resuspended in $M M$ to $\mathrm{OD}_{600}=$ $0.3 \mathrm{CFU} / \mathrm{ml}$, and cultured for different periods before RNA extraction. Primers that were used to amplify probe sequences are listed in Table 2. The PCR products were radio-labeled 
with ${ }^{32} \mathrm{P}$-dCTP using the Random Primed DNA Labeling kit (Ambion, Austin, TX, U.S.A.) as probes.

\section{ChIP and qRT-PCR.}

The ChIP experiments were performed using the ChIP-IT Express kit (Active Motif, Carlsbad, CA, U.S.A.) according to the manufacturer's protocol, and the procedures were modified according to Bruscella and associates (2008). P. syringae bacteria were grown overnight in $\mathrm{KB}$ medium containing gentamicin. Cross-linking was performed by adding formaldehyde (final concentration, 1\%) to the medium for $10 \mathrm{~min}$. The reaction was terminated by adding glycine Stop-Fix solution (Active Motif) and incubating for $10 \mathrm{~min}$ at room temperature with gentle agitation. Bacteria were centrifuged for $1 \mathrm{~min}$ at $12,000 \times g$, washed twice with ice-cold phosphate-buffered saline, resuspended in $1 \mathrm{ml}$ of lysis solution supplemented with $5 \mu \mathrm{l}$ of phenylmethylsulfonyl fluoride (PMSF) and $5 \mu \mathrm{l}$ of protease inhibitor cocktail, and incubated on ice for $30 \mathrm{~min}$. Digestion buffer $(1 \mathrm{ml})$ containing $5 \mu \mathrm{l}$ of PMSF and $5 \mu \mathrm{l}$ of protease inhibitor cocktail was added to the lysate and then heated for $5 \mathrm{~min}$ at $37^{\circ} \mathrm{C}$. An enzymatic shearing mixture (30 $\mu \mathrm{l}$ at $200 \mathrm{U} / \mathrm{ml}$ ) was added to the digestion mixture. After incubation for $25 \mathrm{~min}$ at $37^{\circ} \mathrm{C}$ with agitation, the reaction was stopped by the addition of $20 \mu \mathrm{l}$ of $0.5 \mathrm{M}$ EDTA and incubation for $10 \mathrm{~min}$ on ice. After centrifugation at $15,000 \times g$ and $4^{\circ} \mathrm{C}$ for $10 \mathrm{~min}$, the supernatant was recovered, and the shearing efficiency was examined as described by the manufacturer. Preclearing of chromatin samples, input recovery, immunoprecipitation with or without the anti-HA antibody, addition of Protein $\mathrm{G}$ beads, washing, elution of DNA-protein complexes, reverse cross-linking, RNA removal, and proteinase K treatment were performed by following the manufacturer's instructions.

The eluted DNA samples from the ChIP assay were used for qRT-PCR experiments using Bio-Rad icycler IQ (Bio-Rad, Hercules, CA, U.S.A.). PCR primers (Table 2) for amplification of promoter regions were designed by using the Primer3 software. The PSPTO1489 gene (encoding a putative xenobiotic reductase) that is equally expressed in WT DC3000 and the $\operatorname{rhpS} S^{-}$mutant (L. Lan and X. Tang, unpublished data) was used for normalization. The SYBR green PCR mixture (BioRad) was mixed with appropriate amounts of ChIP samples for qRT-PCR. The amount of PCR product was estimated for different promoter regions using the input DNA (the total sheared DNA prior to ChIP) and the immunoprecipitated DNA with and without the anti-HA antibodies as the matrix. Threshold cycle $(\mathrm{Ct})$ values were obtained for all samples. The ChIP enrichments were determined by the fold change of amplification between the immunoprecipitated DNA with the antibodies (AB) and the immunoprecipitated DNA without the antibodies, and these were calculated by the following formula: $2^{-\Delta \mathrm{Ct}}(\Delta \mathrm{Ct}=$ $\left.\mathrm{Ct}_{\mathrm{AB}}-\mathrm{Ct}_{\mathrm{No} \mathrm{AB}}\right)$. A standard curve and a melt curve were drawn for each primer pair. The slope of the standard curve was used to calculate the primer efficiency for each primer pair. Results were collected only from the reactions showing primer efficiencies between 95 and $105 \%$. A melt curve was drawn for each primer pair to ensure that only one specific PCR product was obtained. The results for all reactions were obtained from at least two independent experiments.

\section{ACKNOWLEDGMENTS}

The work was supported by the United States Department of Agriculture National Research Initiative grants 2003-35319-13246 and 200535319-15299 to X. Tang and the North Central Region Sustainable Agriculture Research and Education Program Graduate Student Grant to X. Deng. Contribution no. 10-119-J from the Kansas Agriculture Experimental Station.

\section{LITERATURE CITED}

Ansaldi, M., Simon, G., Lepelletier, and M., Méjean, V. 2000. The TorR high-affinity binding site plays a key role in both torR autoregulation and torCAD operon expression in Escherichia coli. J. Bacteriol. 182:961-966.

Bang, I. S., Audia, J. P., Park, Y. K., and Foster, J. W. 2002. Autoinduction of the OmpR response regulator by acid shock and control of the Salmonella enterica acid tolerance response. Mol. Microbiol. 44:12351250.

Bijlsma, J. J., and Groisman, E. A. 2003. Making informed decisions: Regulatory interactions between two-component systems. Trends Microbiol. 11:359-366.

Blanco, A. G., Sola, M., Gomis-Rüth, F. X., and Coll, M. 2002. Tandem DNA recognition by $\mathrm{PhoB}$, a two-component signal transduction transcriptional activator. Structure 10:701-713.

Bruscella, P., Eraso, J. M., Roh, J. H., and Kaplan, S. 2008. The use of chromatin immunoprecipitation to define PpsR binding activity in Rhodobacter sphaeroides 2.4.1. J. Bacteriol. 190:6817-6828.

Buell, C. R., Joardar, V., Lindeberg, M., Selengut, J., Paulsen, I. T., Gwinn, M. L., Dodson, R. J., Deboy, R. T., Durkin, A. S., Kolonay, J. F., Madupu, R., Daugherty, S., Brinkac, L., Beanan, M. J., Haft, D. H., Nelson, W. C., Davidsen, T., Zafar, N., Zhou, L., Liu, J., Yuan, Q., Khouri, H., Fedorova, N., Tran, B., Russell, D., Berry, K., Utterback, T., Van Aken, S. E., Feldblyum, T. V., D’Ascenzo, M., Deng, W. L., Ramos, A. R., Alfano, J. R., Cartinhour, S., Chatterjee, A. K., Delaney, T. P., Lazarowitz, S. G., Martin, G. B., Schneider, D. J., Tang, X., Bender, C. L., White, O., Fraser, C. M., and Collmer, A. 2003. The complete genome sequence of the Arabidopsis and tomato pathogen Pseudomonas syringae pv. tomato DC3000. Proc. Natl. Acad. Sci. U.S.A. 100:10181-10186.

Churchward, G. 2007. The two faces of Janus: Virulence gene regulation by CovR/S in group A streptococci. Mol. Microbiol. 64:34-41.

Churchward, G., Bates, C., Gusa, A. A., Stringer, V., and Scott, J. R. 2009. Regulation of streptokinase expression by CovR/S in Streptococcus pyogenes: CovR acts through a single high-affinity binding site. Microbiology. 155:566-575.

Clarke, M. B., and Sperandio, V. 2005. Transcriptional autoregulation by quorum sensing Escherichia coli regulators B and C (QseBC) in enterohaemorrhagic E. coli (EHEC). Mol. Microbiol. 58:441-455.

Dalton, T. L., and Scott, J. R. 2004. CovS inactivates CovR and is required for growth under conditions of general stress in Streptococcus pyogenes. J. Bacteriol. 186:3928-3937.

de Been, M., Bart, M. J., Abee, T., Siezen, R. J., and Francke, C. 2008. The identification of response regulator-specific binding sites reveals new roles of two-component systems in Bacillus cereus and closely related low-GC Gram-positives. Environ. Microbiol. 10:2796-2809.

Deng, X., Xiao, Y., Lan, L., Zhou, J. M., and Tang, X. 2009. Pseudomonas syringae pv. phaseolicola mutants compromising the induction of the type III secretion system. Mol. Plant-Microbe Interact. 22:964-976.

Emmerich, R., Strehler, P., Hennecke, H., and Fischer, H. M. 2000. An imperfect inverted repeat is critical for DNA binding of the response regulator RegR of Bradyrhizobium japonicum. Nucleic Acids Res. 28:4166-4171.

Feil, H., Feil, W. S., Chain, P., Larimer, F., DiBartolo, G., Copeland, A., Lykidis A, Trong, S., Nolan, M., Goltsman, E., Thiel, J., Malfatti, S., Loper, J. E., Lapidus, A., Detter, J. C., Land, M., Richardson, P. M., Kyrpides, N. C., Ivanova, N., and Lindow, S. E. 2005. Comparison of the complete genome sequences of Pseudomonas syringae pv. syringae B728a and pv. tomato DC3000. Proc. Natl. Acad. Sci. U.S.A. 102:11064-11069.

Gao, J., Gusa, A. A., Scott, J. R., and Churchward, G. 2005. Binding of the global response regulator protein CovR to the sag promoter of Streptococcus pyogenes reveals a new mode of CovR-DNA interaction. J. Biol. Chem. 280:38948-38956.

Gonzalo-Asensio, J., Soto, C. Y., Arbués, A., Sancho, J., del Carmen Menéndez, M., García, M. J., Gicquel, B., and Martín, C. 2008. The Mycobacterium tuberculosis phoPR operon is positively autoregulated in the virulent strain H37Rv. J. Bacteriol. 190:7068-7078.

Groisman, E. A. 2001. The pleiotropic two-component regulatory system PhoP-PhoQ. J. Bacteriol. 183:1835-1842.

Guan, K. L., and Dixon, J. E. 1991. Eukaryotic proteins expressed in Escherichia coli: An improved thrombin cleavage and purification procedure of fusion proteins with glutathione S-transferase. Anal. Biochem. 192:262-267.

Gusa, A. A., and Scott, J. R. 2005. The CovR response regulator of group A streptococcus (GAS) acts directly to repress its own promoter. Mol. Microbiol. 56:1195-1207.

Gusa, A. A., Gao, J., Stringer, V., Churchward, G., and Scott, J. R. 2006. Phosphorylation of the group A Streptococcal CovR response regulator 
causes dimerization and promoter-specific recruitment by RNA polymerase. J. Bacteriol. 188:4620-4626.

Gusa, A. A., Froehlich, B. J., Desai, D., Stringer, V., and Scott, J. R. 2007. CovR activation of the dipeptide permease promoter $(P d p p A)$ in group A Streptococcus. J. Bacteriol. 189:1407-1416.

Head, C. G., Tardy, A., and Kenney, L. J. 1998. Relative binding affinities of OmpR and OmpR-phosphate at the $о m p F$ and $о т р C$ regulatory sites. J. Mol. Biol. 281:857-870.

Hendrickson, E. L., Guevera, P., and Ausubel. F. M. 2000. The alternative sigma factor RpoN is required for hrp activity in Pseudomonas syringae pv. maculicola and acts at the level of hrpL transcription. J. Bacteriol. 182:3508-3516.

Hoch, J. A. 2000. Two-component and phosphorelay signal transduction. Curr. Opin. Microbiol. 3:165-170.

Hoffer, S. M., Westerhoff, H. V., Hellingwerf, K. J., Postma, P. W., and Tommassen, J. 2001. Autoamplification of a two-component regulatory system results in "learning" behavior. J. Bacteriol. 183:4914-4917.

Hutchings, M. I., Hong, H. J., and Buttner, M. J. 2006. The vancomycin resistance VanRS two-component signal transduction system of Streptomyces coelicolor. Mol. Microbiol. 59:923-935.

Huynh, T. V., Dahlbeck, D., and Staskawicz, B. J. 1989. Bacterial blight of soybean: Regulation of a pathogen gene determining host cultivar specificity. Science 245:1374-1377.

Joardar, V., Lindeberg, M., Jackson, R. W., Selengut, J., Dodson, R., Brinkac, L. M., Daugherty, S. C., Deboy, R., Durkin, A. S., Giglio, M. G., Madupu, R., Nelson, W. C., Rosovitz, M. J., Sullivan, S., Crabtree, J., Creasy, T., Davidsen, T., Haft, D. H., Zafar, N., Zhou, L., Halpin, R., Holley, T., Khouri, H., Feldblyum, T., White, O., Fraser, C. M., Chatterjee, A. K., Cartinhour, S., Schneider, D. J., Mansfield, J., Collmer, A., and Buell, C. R. 2005. Whole-genome sequence analysis of Pseudomonas syringae pv. phaseolicola 1448A reveals divergence among pathovars in genes involved in virulence and transposition. J. Bacteriol. 187:6488-6498.

King, E. O., Ward, M. K., and Raney, D. E. 1954. Two simple media for the demonstration of pyocyanin and fluorescein. J. Lab. Med. 22:301-307.

Kostakioti, M., Hadjifrangiskou, M., Pinkner, J. S., and Hultgren, S. J. 2009. QseC-mediated dephosphorylation of QseB is required for expression of genes associated with virulence in uropathogenic Escherichia coli. Mol. Microbiol. 73:1020-1031.

Labes, M., Puhler, A., and Simon, R. 1990. A new family of RSF1010derived expression and lac-fusion broad-host-range vectors for gramnegative bacteria. Gene 89:37-46.

Lan, C.-Y., and Igo, M. M. 1998. Differential expression of the OmpF and OmpC porin proteins in Escherichia coli $\mathrm{K}-12$ depends upon the level of active OmpR. J. Bacteriol. 180:171-174.

Lan, L., Deng, X., Zhou, J. M, and Tang, X. 2006. Genome-wide gene expression analysis of Pseudomonas syringae pv. tomato DC3000 reveals overlapping and distinct pathways regulated by $h r p L$ and $h r p R S$.
Mol. Plant-Microbe Interact. 19:976-987.

Laub, M. T., and Goulian, M. 2007. Specificity in two-component signal transduction pathways. Annu. Rev. Genet. 41:121-145.

Maris, A. E., Sawaya, M. R., Kaczor-Grzeskowiak, M., Jarvis, M. R., Bearson, S. M., Kopka, M. L., Schröder, I., Gunsalus, R. P., and Dickerson, R. E. 2002. Dimerization allows DNA target site recognition by the NarL response regulator. Nat. Struct. Biol. 9:771-778.

Maris, A. E., Walthers, D., Mattison, K., Byers, N., and Kenney, L. J. 2005. The response regulator OmpR oligomerizes via beta-sheets to form head-to-head dimers. J. Mol. Biol. 350:843-856.

McCleary, W. R., Stock, J. B., and Ninfa, A. J. 1993. Is acetyl phosphate a global signal in Escherichia coli? J. Bacteriol. 175:2793-2798.

Shin, D., Lee, E. J., Huang, H., and Groisman, E. A. 2006. A positive feedback loop promotes transcription surge that jump-starts Salmonella virulence circuit. Science 314:1607-1609.

Soncini, F. C., Véscovi, E. G., and Groisman, E. A. 1995. Transcriptional autoregulation of the Salmonella typhimurium phoPQ operon. J. Bacteriol. 177:4364-4371.

Stock, A. M., Robinson, V. L., and Goudreau, P. N. 2000. Two-component signal transduction. Annu. Rev. Biochem. 69:183-215.

Tang, X., Xiao, Y., and Zhou, J.-M. 2006. Regulation of the type III secretion system in phytopathogenic bacteria. Mol. Plant-Microbe Interact. 19:1159-1166.

Xiao, F., Goodwin, S. M, Xiao, Y., Sun, Z., Baker, D., Tang, X., Jenks, M. A., and Zhou, J. M. 2004. Arabidopsis CYP86A2 represses Pseudomonas syringae type III genes and is required for cuticle development. EMBO (Eur. Mol. Biol. Organ.) J. 23:2903-2913.

Xiao, Y., and Hutcheson S. W. 1994. A single promoter sequence recognized by a newly identified alternate sigma factor directs expression of pathogenicity and host range determinants in Pseudomonas syringae. J. Bacteriol. 176:3089-3091.

Xiao, Y., Heu, S., Yi, J., Lu, Y., and Hutcheson, S. W. 1994. Identification of a putative alternate sigma factor and characterization of a multicomponent regulatory cascade controlling the expression of Pseudomonas syringae pv. syringae Pss61 hrp and hrmA genes. J. Bacteriol. 176:1025-1036.

Xiao, Y., Lan, L., Yin, C., Deng, X., Baker, D., Zhou, J. M., and Tang, X. 2007. Two-component sensor RhpS promotes induction of Pseudomonas syringae type III secretion system by repressing negative regulator RhpR. Mol. Plant-Microbe Interact. 20:223-234.

Yoshida, T., Qin, L., Egger, L.A., and Inouye, M. 2006. Transcription regulation of $o m p F$ and $o m p C$ by a single transcription factor, OmpR. J. Biol. Chem. 281:17114-17123.

\section{AUTHOR-RECOMMENDED INTERNET RESOURCE}

Primer3 server: frodo.wi.mit.edu 